TRANSACTIONS OF THE

AMERICAN MATHEMATICAL SOCIETY

Volume 364, Number 12, December 2012, Pages 6371-6394

S 0002-9947(2012)05602-9

Article electronically published on July 11, 2012

\title{
COHEN-MACAULAY RESIDUAL INTERSECTIONS AND THEIR CASTELNUOVO-MUMFORD REGULARITY
}

\author{
SEYED HAMID HASSANZADEH
}

\begin{abstract}
In this article we study the structure of residual intersections via constructing a finite complex of not necessarily free modules. The complex provides information about an ideal which coincides with the residual intersection in the geometric case; and is closely related to it in general. A new success obtained through studying such a complex is to prove the Cohen-Macaulayness of residual intersections of a wide class of ideals. In particular, it is shown that in a Cohen-Macaulay local ring any geometric residual intersection of an ideal which satisfies the sliding depth condition is Cohen-Macaulay. This is an affirmative answer for one of the main open questions in the theory of residual intersections (Huneke and Ulrich, 1988, Question 5.7).

The complex that we come up with in this article suffices to obtain a bound for the Castelnuovo-Mumford regularity of a residual intersection in terms of the degrees of minimal generators. More precisely, in a positively graded Cohen-Macaulay *local ring $R=\bigoplus_{n \geq 0} R_{n}$, if $J=\mathfrak{a}: I$ is a "geometric" $s$-residual intersection such that $\operatorname{Ht}(I)=g>0$ and $I$ satisfies a sliding depth condition, then $\operatorname{reg}(R / J) \leq \operatorname{reg}(R)+\operatorname{dim}\left(R_{0}\right)+\sigma(\mathfrak{a})-(s-g+1) \operatorname{indeg}(I / \mathfrak{a})-s$, where $\sigma(\mathfrak{a})$ is the sum of the degrees of elements of a minimal generating set of $\mathfrak{a}$. It is also shown that the equality holds whenever $I$ is a perfect ideal of height 2 and $R_{0}$ is a field.
\end{abstract}

\section{INTRODUCTION}

The notion of the residual intersection was originally introduced by Artin and Nagata [1]; it has been extensively studied by Huneke, Ulrich and others. Residual intersections classify interesting classes of ideals in graded and local rings and have significant geometric applications [10].

Throughout the paper, $R$ is a Noetherian (graded) ring. Let $I$ be a (graded) ideal of height $g$ in the local (*local) ring $R$, and let $s \geq g$ be an integer. An $s$-residual intersection of $I$ is an ideal $J$ such that $J=\mathfrak{a}: I$ for some (graded) ideal $\mathfrak{a} \subseteq I$ with $\operatorname{ht}(J) \geq s \geq \mu(\mathfrak{a})$ ( $\mu$ denoting the minimal number of generators). In the case where $R$ is Gorenstein, $I$ is unmixed, and ht $(I)=\operatorname{ht}(J)$, the notions of residual intersection and linkage are the same. Two important examples of residual intersections which also demonstrate the ubiquity of such ideals are as follows (these examples are given in [18,4.1-4.3]): The ideal defined by the maximal minors of a generic $s$ by $r$ matrix with $r<s$ is an $(s-r+1)$-residual intersection of the ideal defined by the maximal minors of a generic $s \times(s+1)$ matrix, which is a perfect ideal of height 2. Another example is if $R$ is a Cohen-Macaulay (from now

Received by the editors June 18, 2009 and, in revised form, December 31, 2010.

2010 Mathematics Subject Classification. Primary 13C40, 13D02, 13H10; Secondary 14C17.

Key words and phrases. Castelnuovo-Mumford regularity, generalized Koszul complex, $G_{S}$ condition, residual intersection, sliding depth condition.

(C)2012 American Mathematical Society Reverts to public domain 28 years from publication 
on, abbreviated by $\mathrm{CM}$ ) local ring and $I$ is an ideal of positive height that satisfies the condition $G_{\infty}$, then the defining ideal of the extended symmetric algebra of $I$ is a residual intersection.

A main problem in the context of residual intersections is to find conditions when residual intersections are Cohen-Maucaulay, since this is a central property that controls various invariants of ideals. The CM property and the structure of canonical module of residual intersections are carefully studied in several works; e.g., 7, 18, 19, 30. Most of these works deeply apply a crucial lemma of Artin and Nagata [1, Lemma 2.3] which provides an inductive argument to reduce a problem in residual intersections to a similar problem in the linkage theory. One of the most important conditions required for this lemma, or similar results, is the $G_{s}$ condition which bounds the local number of generators of an ideal. More precisely, we say that an ideal $I$ satisfies the condition $G_{s}$ if $\mu\left(I_{\mathfrak{p}}\right) \leq \mathrm{ht}(\mathfrak{p})$ for all prime ideal $\mathfrak{p}$ containing $I$ such that $h t(\mathfrak{p}) \leq s-1$. We say that $I$ satisfies $G_{\infty}$ if $I$ satisfies $G_{s}$ for all $s$. The other conditions which are required to provide the CM property of residual intersections are some depth conditions on Koszul homology modules of $I$ such as strongly Cohen-Macaulay (SCM) and sliding depth condition (SD). Another goal in the theory of residual intersections is to find the entire free resolution or even the generators of residual intersections. An explicit resolution for residual intersections is only known in some special cases and involves generalized Koszul complexes and approximation complexes; e.g., 4] and [22].

The interplay among residual intersections and some arithmetic subjects in commutative algebra such as analytic spread, reduction number, etc. (see [17, [27, 32]) is at the origin of many attempts to weaken the conditions which infer some arithmetic properties of residual intersections. Some sort of depth assumption was known to be required for residual intersections to be Cohen-Macaulay; see [30. On the other hand, removing the $G_{s}$ condition remained as the main challenge in the theory of residual intersections. As C. Huneke and B. Ulrich mentioned in their paper [19, Question 5.7], the main open question is:

\section{Suppose that $R$ is a local CM ring and $I$ is an ideal of $R$ which is $S C M$ (or even has sliding depth). Let $J$ be any residual intersection of $I$. Then is $R / J$ CM?}

One main purpose of this paper is to answer this question affirmatively. The idea is that we construct a finite complex $\mathcal{C}$. whose tail consists of free modules and whose beginning terms are finite direct sums of cycles of the Koszul complex. It is shown in Proposition 2.8 that this complex is acyclic under some sliding depth conditions on cycles of the Koszul complex. These conditions are precisely defined in Definition 2.3 with the abbreviated form $\mathrm{SDC}_{k}$ for some integer $k$. We then provide some conditions which imply the $\mathrm{SDC}_{k}$ condition. By the way, in Proposition 2.6. we completely determine the local cohomology modules (and consequently clarify the depth) of the last cycle of the Koszul complex wherein the Koszul homology is not zero. This result improves a proposition of Herzog, Vasconcelos and Villareal [16. 1.1]. This investigation ensures the acyclicity of the complex $\mathcal{C}$. without any assumption on $I$ whenever the residual intersection is close to the linkage, i.e., when $s-g \leq 2$; see Corollary 2.9. The importance of $s$-residual intersections which are close to linked ideals is due to the fact that these ideals contain a class of ideals whose Rees algebras are CM; see for example [17] and [30. The ideal which is resolved by $\mathcal{C}_{\bullet}$, say $K$, is quite close to the residual intersection. Indeed, in Theorem 
2.11 it is shown that $K$ is always contained in $J$ and has the same radical as $J$. Moreover, if $I$ satisfies the sliding depth condition $\mathrm{SDC}_{1}$, then $K$ is CM. Therefore, the affirmative answer for the above-mentioned open question is in the case where $K=J$. It is shown in Theorem 2.11(iv) that if $I / \mathfrak{a}$ is generated by at most one element locally in height $s$, then $K=J$. In particular, if the residual intersection is geometric, then the answer to the "question" is affirmative; see Corollary 2.12.

Having an approximation complex for the residual intersection in hand, we establish a bound for the Castelnuovo-Mumford regularity of residual intersections in terms of the degrees of their defining ideals. Determining this bound needs several careful studies of the degrees and the maps in $\mathcal{C}_{\bullet}$. More precisely, it is shown in Theorem 3.6 that if $J=\mathfrak{a}: I$ is an $s$-residual intersection of an ideal $I$ that satisfies the $\mathrm{SD}_{1}$ condition with $\mathrm{ht}(I)=g>0$ and $I / \mathfrak{a}$ is generated by at most one element locally in height $s$, then

$$
\operatorname{reg}(R / J) \leq \operatorname{reg}(R)+\operatorname{dim}\left(R_{0}\right)+\sigma(\mathfrak{a})-(s-g+1) \operatorname{indeg}(I / \mathfrak{a})-s .
$$

This formula generalizes the previous known facts about the regularity of linked ideals. With the course of the proof of Theorem 3.6, we need to know about the relation between the ordinary Castelnuovo-Mumford regularity of finitely generated graded $R$-modules and another invariant which we call the regularity with respect to the maximal ideal, $\operatorname{reg}_{\mathfrak{m}}(M):=\max \left\{\operatorname{end}\left(H_{\mathfrak{m}}^{i}(M)\right)+i\right\}$. It is shown in Proposition 3.4 that

$$
\operatorname{reg}(M) \leq \operatorname{reg}_{\mathfrak{m}}(M) \leq \operatorname{reg}(M)+\operatorname{dim}\left(R_{0}\right)
$$

This proposition enables us to state the above inequality for the regularity of residual intersection without any restriction on the dimension of $R_{0}$. Besides, it generalizes previous results of Hyry [21] and Trung [29].

In the presence of the $G_{s}$ condition, in Lemma 3.11, we prove a graded version of the crucial lemma of Artin and Nagata. With the aid of this lemma, under the condition $G_{s}$, in Proposition 3.14, a different proof (from Theorem 3.6) for the inequality of the regularity of residual intersections is given. In addition, a criterion is provided to decide when the regularity of residual intersections attains the proposed upper bound.

The paper is closed with the formula of the regularity of the residual intersections of perfect ideals of height 2 .

Some of the straightforward verifications which are omitted in the proofs can be found in the author's Ph.D. thesis.

\section{Residual intersection without the $G_{s}$ COndition}

Throughout this section $R$ is a Noetherian ring of dimension $d, I=\left(f_{1}, \cdots, f_{r}\right)$ is an ideal of grade $g \geq 1, \mathfrak{a}=\left(l_{1}, \cdots, l_{s}\right)$ is an ideal contained in $I, s \geq g, J=\mathfrak{a}:_{R} I$, and $S=R\left[T_{1}, \cdots, T_{r}\right]$ is a polynomial extension of $R$ with indeterminates $T_{i}$ 's. We denote the symmetric algebra of $I$ over $R$ by $\mathcal{S}_{I}$ and consider $\mathcal{S}_{I}$ as an $S$-algebra via the ring homomorphism $S \rightarrow \mathcal{S}_{I}$ sending $T_{i}$ to $f_{i}$ as an element of $\left(\mathcal{S}_{I}\right)_{1}=I$. Let $\left\{\gamma_{1}, \cdots, \gamma_{s}\right\} \subseteq S_{1}$ be linear forms whose images under the above homomorphism are $l_{i} \in\left(\mathcal{S}_{I}\right)_{1}$, let $(\gamma)$ be the $S$-ideal generated by $\gamma_{i}$ 's and let $\mathfrak{g}:=\left(T_{1}, \cdots, T_{r}\right)$. For a sequence of elements $\mathfrak{x}$ in a commutative $\operatorname{ring} A$ and an $A$-module $M$, we denote the koszul complex by $K_{\bullet}(\mathfrak{x} ; M)$, its cycles by $Z_{i}(\mathfrak{x} ; M)$ and homologies by $H_{i}(\mathfrak{x} ; M)$. For a graded module $M, \operatorname{indeg}(M):=\inf \left\{i: M_{i} \neq 0\right\}$ and $\operatorname{end}(M):=\sup \left\{i: M_{i} \neq 0\right\}$. Setting $\operatorname{deg}\left(T_{i}\right)=1$ for all $i, S$ is a standard graded over $S_{0}=R$. 
To set one more convention, when we draw the picture of a double complex obtained from a tensor product of two finite complexes (in the sense of [33, 2.7.1]), say $\mathcal{A} \otimes \mathcal{B}$, we always put $\mathcal{A}$ in the vertical direction and $\mathcal{B}$ in the horizontal one. We also label the module which is in the upper-right corner by $(0,0)$ and consider the labels for the rest, as the points in the third quadrant.

Now consider the koszul complex

$$
K_{\bullet}(f ; R): 0 \rightarrow K_{r} \stackrel{\delta_{r}^{f}}{\longrightarrow} K_{r-1} \stackrel{\delta_{r-1}^{f}}{\longrightarrow} \cdots \rightarrow K_{0} \rightarrow 0 .
$$

Let $Z_{i}=Z_{i}(f ; R)$. The $\mathcal{Z}$-complex of $I$ with coefficients in $R$ is

$$
\begin{aligned}
\mathcal{Z}_{\bullet}= & \mathcal{Z}_{\bullet}(f ; R): 0 \rightarrow Z_{r-1} \otimes_{R} S(-r+1) \stackrel{\delta_{r-1}^{T}}{\longrightarrow} \cdots \rightarrow Z_{1} \otimes_{R} S(-1) \\
& \stackrel{\delta_{1}^{T}}{\longrightarrow} Z_{0} \otimes_{R} S \rightarrow 0 .
\end{aligned}
$$

Recall that $H_{0}\left(\mathcal{Z}_{\bullet}\right)=\mathcal{S}_{I}$ and $H_{i}\left(\mathcal{Z}_{\bullet}\right)$ is a finitely generated $\mathcal{S}_{I}$-module for all $i$, 14, 4.3] and notice that $Z_{r}=0$ because of the standing assumption $g \geq 1$.

In order to make our future structures and computations more transparent, we need to add some intricacies to the $\mathcal{Z}$-complex.

For $i \geq r-g+1$, the tail of the koszul complex $K_{\bullet}(f ; R)$ resolves $Z_{i}$. Now we construct our first double complex $\mathcal{F}$ with $\mathcal{F}_{-i,-j}=K_{r-j+i} \otimes_{R} S(-i-r+g-1)$ for $0 \leq i \leq g-2$ and $0 \leq j \leq g-i-2 . \mathcal{F}$ is a truncation of $K_{\bullet}(f ; R) \otimes_{R} K_{\bullet}(T ; S)$ : $(\delta:=-r+g-1)$

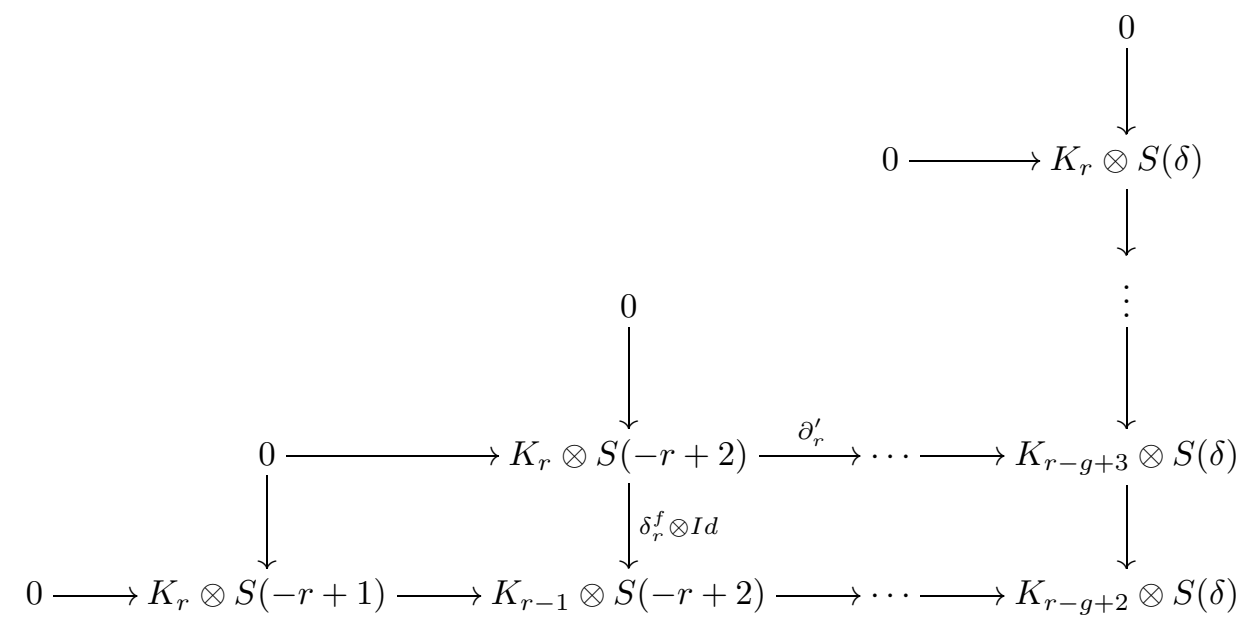

The complex $\mathcal{F}$ is a double complex of free $S$-modules which maps vertically onto the tail of $\mathcal{Z}_{\bullet}$. Therefore, if we replace the last $g$ modules of $\mathcal{Z}_{\bullet}$ by $\operatorname{Tot}(\mathcal{F})$, with the composition map

$$
\begin{aligned}
K_{r-g+2} \otimes_{R} S(-r+g-1) & \stackrel{\delta_{r-g+2}^{f} \otimes I d}{\longrightarrow} Z_{r-g+1} \otimes_{R} S(-r+g-1) \\
& \stackrel{\delta_{r-g+1}^{T}}{\longrightarrow} Z_{r-g} \otimes_{R} S(-r+g),
\end{aligned}
$$

then we have a modified $\mathcal{Z}$-complex, say $\mathcal{Z}_{\bullet}^{\prime}$, which has the same homologies as $\mathcal{Z}_{\bullet}$ (see [12]), while its tail consists of free $S$-modules. Precisely,

$$
\mathcal{Z}_{\bullet}^{\prime}:=0 \rightarrow \mathcal{Z}_{r-1}^{\prime} \rightarrow \cdots \rightarrow \mathcal{Z}_{0}^{\prime} \rightarrow 0
$$


where

$$
\mathcal{Z}_{i}^{\prime}=\left\{\begin{array}{cl}
K_{i+1} \otimes_{R}\left(\bigoplus_{t=r-i}^{g-1} S(-r-t)\right) & \text { if } i \geq r-g+1 \\
\mathcal{Z}_{i} & \text { otherwise. }
\end{array}\right.
$$

Now consider the double complex $\mathcal{E}:=\mathcal{Z}^{\prime} \bullet \otimes_{S} K_{\bullet}(\gamma ; S)$. Denote $\mathcal{D}_{\bullet}:=\operatorname{Tot}(\mathcal{E})$ as the following complex:

$$
\mathcal{D}_{\bullet}: 0 \rightarrow D_{r+s-1} \rightarrow \cdots \rightarrow D_{1} \rightarrow D_{0} \rightarrow 0 \text {. }
$$

Then $H_{0}\left(\mathcal{D}_{\bullet}\right)=\mathcal{S}_{I} /(\gamma) \mathcal{S}_{I}$, and for all $0 \leq i \leq r+s-1$ the largest integer $i$ such that $S(-i)$ appears in the summands of $D_{i}$ is $i$. Moreover,

$$
\operatorname{indeg}\left(D_{i}\right)=\left\{\begin{array}{cl}
i, & 0 \leq i \leq r-g \\
r-g+1, & r-g+1 \leq i \leq r-1, \\
i-g+2, & r \leq i \leq r+s-1
\end{array}\right.
$$

Still, we want to study the properties of the complex $\mathcal{D}_{\bullet}$. We shall sometimes use the following lemma.

Lemma 2.1. Let $M$ be an R-module. Then

(i) $H_{\mathfrak{g}}^{i}\left(M \otimes_{R} S\right)=0$ for all $i \neq r$,

(ii) there exists a functorial isomorphism $\theta_{M}: H_{\mathfrak{g}}^{r}\left(M \otimes_{R} S\right) \rightarrow M \otimes_{R} H_{\mathfrak{g}}^{r}(S)$.

Proof (see [11, 2.1.11]). The proof follows along the same line as in the case $M=$ $R$. (i) follows from the fact that $T_{1}, \cdots, T_{r}$ is a regular sequence on $M \otimes_{R} S$ and (ii) from the computation of $H_{\mathfrak{g}}^{r}(-)$ via the Cech complex on $T_{1}, \cdots, T_{r}$.

The above lemma implies that $H_{\mathfrak{g}}^{j}\left(D_{i}\right)=0$ if $j \neq r$ and end $\left(H_{\mathfrak{g}}^{r}\left(D_{i}\right)\right) \leq-r+i$ for all $i$. In particular, $H_{\mathfrak{g}}^{r}\left(D_{i}\right)_{0}=0$ for all $i \leq r-1$. In the spirit of [5, 3.2(iv)] we introduce the complex $\mathcal{Z}_{\bullet}^{+}$of $R$-modules,

$$
\mathcal{Z}_{\bullet}^{+}:=H_{\mathfrak{g}}^{r}\left(\mathcal{D}_{\bullet}\right)_{0}: 0 \rightarrow Z_{r-1}^{+} \rightarrow \cdots \rightarrow Z_{r-s+1}^{+} \stackrel{\varphi_{0}}{\rightarrow} Z_{r-s}^{+} \rightarrow 0
$$

Notice that $\mathcal{Z}_{i}^{+}=Z_{r-s+i}^{+}$, where, by Lemma 2.1, for $j \geq r-g+1, Z_{j}^{+}$is a free $R$-module and for $j \leq r-g$ it is a direct sum of finitely many copies of some elements of the set $\left\{Z_{\max \{j, 0\}}, \cdots, Z_{r-1}\right\}$.

M. Chardin and B. Ulrich [5, 3.2] show that under some conditions on $I$ and $\mathfrak{a}$ the only non-zero homology of this complex is Coker $\varphi_{0} \cong \mathfrak{a}: I$. Our aim in this section is to extend their result by removing almost all of the conditions imposed on $I$ and $\mathfrak{a}$ to obtain a sufficient condition for the acyclicity of $\mathcal{Z}_{\bullet}^{+}$and to determine the structure of Coker $\varphi_{0}$. Achieving this aim sheds some light on the structure of residual intersections. The next lemma is a key to our aim.

Lemma 2.2. If $I=\mathfrak{a}$, then the only non-zero homology of $\mathcal{Z}_{\bullet}^{+}$is Coker $\varphi_{0} \cong R$.

Proof. Let $\mathcal{C}_{\mathfrak{g}}^{\bullet}(S)$ be the Čech complex associated to $\mathfrak{g}$ and $S$. Consider the double complex $\mathcal{G}:=\mathcal{C}_{\mathfrak{g}}^{\bullet}(S) \otimes_{S} \mathcal{D}$. By Lemma 2.1, all the vertical homologies except those in the base row vanish. Therefore

$$
{ }^{1} E_{\text {ver }}=0 \rightarrow H_{\mathfrak{g}}^{r}\left(D_{r+s-1}\right) \rightarrow \cdots \rightarrow H_{\mathfrak{g}}^{r}\left(D_{r+1}\right) \stackrel{\varphi}{\rightarrow} H_{\mathfrak{g}}^{r}\left(D_{r}\right) \rightarrow \cdots \rightarrow H_{\mathfrak{g}}^{r}\left(D_{0}\right) \rightarrow 0 .
$$

By definition $\left({ }^{1} E_{\text {ver }}\right)_{0}=\mathcal{Z}_{\bullet}^{+}$.

Now we return to the (third quadrant) double complex $\mathcal{E}$ with $\mathcal{D} \bullet:=\operatorname{Tot}(\mathcal{E})$ in the case where $I=\mathfrak{a}$. The vertical spectral sequence arising from $\mathcal{E}$ at point $(-i,-j)$ has as the first term $H_{j}\left(\mathcal{Z}_{\bullet}^{\prime} \otimes_{S} \bigwedge^{i} S(-1)^{s}\right) \cong H_{j}\left(\mathcal{Z}_{\bullet}\right) \otimes_{S} \bigwedge^{i} S(-1)^{s}$. As $H_{j}\left(\mathcal{Z}_{\bullet}\right)$ is an $\mathcal{S}_{I^{-}}$-module, it then follows that $H_{i}\left(\mathcal{D}_{\bullet}\right)$, for all $i$, is annihilated by a 
power of $L=\operatorname{ker}\left(S \rightarrow \mathcal{S}_{I}\right)$. Since $I=\mathfrak{a}, \mathfrak{g}=\mathfrak{g}+L=(\gamma)+L$, hence $H_{\mathfrak{g}}^{j}\left(H_{i}\left(\mathcal{D}_{\bullet}\right)\right)=$ $H_{(\gamma)}^{j}\left(H_{i}\left(\mathcal{D}_{\bullet}\right)\right)$ for all $i$ and $j$. On the other hand, the horizontal spectral sequence (arising from $\mathcal{E}$ ) at the point $(-i,-j)$ has as the first term $H_{i}\left((\gamma) ; \mathcal{Z}_{j}^{\prime}\right)$ which is annihilated by $(\gamma)$. Therefore the convergence of the horizontal spectral sequence to the homology modules of $\mathcal{D}_{\bullet}$ implies that $H_{i}\left(\mathcal{D}_{\bullet}\right)$ is annihilated by some powers of $(\gamma)$ for all $i$. Hence $H_{\mathfrak{g}}^{j}\left(H_{i}\left(\mathcal{D}_{\bullet}\right)\right)=H_{(\gamma)}^{j}\left(H_{i}\left(\mathcal{D}_{\bullet}\right)\right)=0$ for all $j \geq 1$ and all $i$. Moreover, we have indeg $\left(H_{\mathfrak{g}}^{0}\left(H_{i}\left(\mathcal{D}_{\bullet}\right)\right)\right) \geq \operatorname{indeg}\left(D_{i}\right) \geq 1$ for $i \geq 1$.

Summing up the above paragraph, the second horizontal spectral sequence associated to $\mathcal{G}$ is

$$
\left({ }^{2} E_{\text {hor }}^{-i,-j}\right)_{0}=H_{\mathfrak{g}}^{j}\left(H_{i}\left(\mathcal{D}_{\bullet}\right)\right)_{0}=\left\{\begin{array}{cl}
H_{(\gamma)}^{0}\left(H_{0}(\mathcal{D} \bullet)\right)_{0} & \text { if } i=j=0 \\
0 & \text { otherwise }
\end{array}\right.
$$

Now the acyclicity of $\mathcal{Z}_{\bullet}^{+}$and the identification Coker $\varphi_{0} \cong H_{\gamma}^{0}\left(\mathcal{S}_{I} /(\gamma) \mathcal{S}_{I}\right)_{0}=$ $\left(\mathcal{S}_{I} /(\gamma) \mathcal{S}_{I}\right)_{0}=R$ comes from the fact that ${ }^{2} E_{\text {ver }}^{-i,-j}={ }^{\infty} E_{\text {ver }}^{-i,-j}$ for all $i, j$ and the above computation for $\left({ }^{2} E_{\text {hor }}^{-i,-j}\right)_{0}$.

The concept of the sliding depth condition SD first appeared in the study of the acyclicity of some approximation complexes by Herzog, Simis and Vasconcelos in [14. This concept was then formally defined by the same authors in 15. Let $k$ and $t$ be two integers; we say that the ideal $I$ satisfies $\mathrm{SD}_{k}$ at level $t$ if $\operatorname{depth}\left(H_{i}(f ; R)\right) \geq$ $\min \{d-g, d-r+i+k\}$ for all $i \geq r-g-t$ (whenever $t=r-g$ we simply say that $I$ satisfies $\mathrm{SD}_{k}$, also $\mathrm{SD}$ stands for $\mathrm{SD}_{0}$ ). However, for our purposes in this paper, we need a slightly weaker condition than the sliding depth condition.

Definition 2.3. Let $k$ and $t$ be two integers. We say that $I$ satisfies the sliding depth condition on cycles, $\mathrm{SDC}_{k}$, at level $t$, if

$$
\operatorname{depth}\left(Z_{i}\right) \geq \min \{d-r+i+k, d-g+2, d\}
$$

for all $r-g-t \leq i \leq r-g$.

Remark 2.4. We make several observations on the basic properties of the condition SDC in the case where $R$ is a CM local ring (see [12] for some details).

(i) The property $\mathrm{SDC}_{k}$ at level $t$ localizes, and it only depends on $I, 31$.

(ii) $\mathrm{SD}_{k}$ implies $\mathrm{SDC}_{k+1}$; see Proposition 2.5

(iii) Whenever $\operatorname{depth}(R) \geq 2 \operatorname{depth}\left(Z_{i}(f ; R)\right) \geq 2$ for all $i$. Furthermore, if $I \neq R$, for all $r-1 \geq i \geq r-g+1, Z_{i}$ is a module of finite projective dimension $r-i-1$. Hence $\operatorname{depth}\left(Z_{i}\right)=d-(r-i-1)=d-r+i+1$, for all $r-1 \geq i \geq r-g+1$.

(iv) If $\operatorname{depth}\left(\operatorname{Ext}_{R}^{i}(R / I, R)\right) \geq d-i-1$ for all $i \geq g+1$, for example if $R$ is Gorenstein and $I$ is CM, then it is not difficult to deduce that $H_{r-g}(f ; R)$ is $\mathrm{CM}$ of dimension $d-g$. In this case one can see from the exact sequence $0 \rightarrow B_{r-g}(f ; R) \rightarrow Z_{r-g} \rightarrow H_{r-g}(f ; R) \rightarrow 0$ that $\operatorname{depth}\left(Z_{r-g}\right) \geq d-g$. Therefore, in this case, $I$ satisfies $\mathrm{SDC}_{0}$ at level 0 .

(v) In the case where $R$ is Gorenstein local and $I^{u n m}$ is CM, where $I^{u n m}$ is the unmixed part of $I$, it is shown in Proposition 2.6 that $I$ satisfies $\mathrm{SDC}_{1}$ at level $0 . \mathrm{SDC}_{1}$ at level +1 is more mysterious; see Example 2.10.

Proposition 2.5. $\mathrm{SD}_{k}$ implies $\mathrm{SDC}_{k+1}$, whenever $R$ is a $C M$ local ring. 
Proof. Consider the truncated Koszul complex

$$
0 \rightarrow Z_{i} \rightarrow K_{i} \rightarrow K_{i-1} \rightarrow \cdots \rightarrow K_{0} \rightarrow 0 .
$$

Tensoring the Cech complex, $\mathcal{C}_{\mathfrak{m}}^{\bullet}(R)$, with this complex, we have the following spectral sequences:

$$
{ }^{1} E_{\text {ver }}^{-p,-q}=\left\{\begin{array}{cc}
H_{\mathfrak{m}}^{q}\left(Z_{i}\right), & p=i+1, \\
0, & p \neq i+1 \text { and } q \neq d, \\
H_{\mathfrak{m}}^{d}\left(K_{p}\right), & p \neq i+1 \text { and } q=d,
\end{array}\right.
$$

so that ${ }^{1} E_{\text {ver }}^{-p,-q} \cong{ }^{2} E_{\text {ver }}^{-p,-q}$ for all $q \neq d$, and ${ }^{2} E_{\text {ver }}^{-p,-q}={ }^{\infty} E_{\text {ver }}^{-p,-q}$ for any $p$ and $q$. Recall that $\mathrm{SDC}_{k+1}$ is equivalent to saying that ${ }^{1} E_{v e r}^{-p,-q}=0$ for $p=i+1, i \leq r-g$ and $q \leq \min \{d-r+k+p-1, d-g+1, d-1\}$.

On the other hand,

$$
{ }^{2} E_{\text {hor }}^{-p,-q}= \begin{cases}0, & p \geq i, \text { or } p \geq r-g-k \text { and } q \leq d-g-1, \\ 0, & p \leq \min \{i-1, r-g-k-1\} \text { and } q-p \leq d-r+k-1,\end{cases}
$$

so that the result follows from the convergence of the spectral sequences.

Recall that the unmixed part of an ideal $I, I^{u n m}$, is the intersection of all primary components of $I$ with height equal to ht $(I)$. If $I^{\prime}$ is an ideal that coincides with $I$ locally in height ht $(I)$ in $\mathrm{V}(I)$, then $I^{\prime} \subseteq I^{u n m}$, 24, Exercise 6.4]. Also $I^{\text {unm }} \subseteq \operatorname{Ann}\left(H_{r-g}(f ; R)\right)=\operatorname{Ann}\left(\operatorname{Ext}_{R}^{g}(R / I, R)\right)$, and the equality holds if $R$ is Gorenstein locally in height ht $(I)$. Recall that if $R$ is Gorenstein local, then $\omega_{R / I}:=\operatorname{Ext}_{R}^{g}(R / I, R)$ is called the canonical module of $R / I$ in the sense of [13].

In [16, 1.1], Herzog, Vasconcelos and Villarreal present a lower bound for $\operatorname{depth}\left(Z_{r-g}\right)$ in the case where $R$ is Gorenstein local and $I$ is CM. In the next proposition we clarify all of the local cohomology modules of $Z_{r-g}$ and determine exactly $\operatorname{depth}\left(Z_{r-g}\right)$, which gives a complete generalization to [16, 1.1].

Proposition 2.6. Suppose that $(R, \mathfrak{m})$ is Gorenstein and denote by ${ }^{v}$ the Matlis dual. Then

(i) $H_{\mathfrak{m}}^{i}\left(Z_{r-g}\right) \cong H_{\mathfrak{m}}^{i}\left(\omega_{R / I}\right)$ for $i<d-g$,

(ii) $H_{\mathfrak{m}}^{d-g}\left(Z_{r-g}\right) \cong\left(\operatorname{Coker}\left(R / I \stackrel{\text { can. }}{\longrightarrow} \operatorname{End}_{R}\left(\omega_{R / I}\right)\right)\right)^{v}$,

(iii) $H_{\mathfrak{m}}^{d-g+1}\left(Z_{r-g}\right) \cong\left(I^{u n m} / I\right)^{v}$ whenever $g \geq 2$,

(iv) $H_{\mathfrak{m}}^{d-g+i}\left(Z_{r-g}\right) \cong\left(H_{i-1}(f ; R)\right)^{v}$ for $2 \leq i \leq g-1$,

(v) $H_{\mathfrak{m}}^{d}\left(Z_{r-g}\right) \cong\left(I^{u n m}\right)^{v}$, if $g=1$.

In particular, either $\operatorname{depth}\left(Z_{r-g}\right)=\operatorname{depth}\left(\omega_{R / I}\right)$ or $R / I^{\text {unm }}$ is $C M$. In the latter case

(1) $\operatorname{depth}\left(Z_{r-g}\right)=d$ if either $g=1$ or $f$ is a regular sequence.

(2) $\operatorname{depth}\left(Z_{r-g}\right)=d-g+1$ if $I$ is not unmixed.

(3) $\operatorname{depth}\left(Z_{r-g}\right)=d-g+2$ if $g \geq 2, I$ is $C M$ and $f$ is not a regular sequence.

Proof. Consider the short exact sequence $0 \rightarrow B_{r-g} \rightarrow Z_{r-g} \rightarrow \omega_{R / I}\left(\cong H_{r-g}(f ; R)\right)$ $\rightarrow 0$. Since $B_{r-g}$ is a module of projective dimension $g-1$, depth $B_{r-g}=d-g+$ 1 and $\operatorname{Ext}_{R}^{i}\left(Z_{r-g}, R\right) \cong \operatorname{Ext}_{R}^{i}\left(\omega_{R / I}, R\right)$ for $i \geq g+1$. Now (i) follows by the local duality. Since $\operatorname{Ext}_{R}^{i}\left(\omega_{R / I}, R\right)=0$ for $i \leq g-1$, we have $\operatorname{Ext}_{R}^{g-i}\left(Z_{r-g}, R\right)=$ $\operatorname{Ext}_{R}^{g-i}\left(B_{r-g}, R\right)$ for all $2 \leq i \leq g$, and the following sequence is exact:

$$
0 \rightarrow \operatorname{Ext}_{R}^{g-1}\left(Z_{r-g}, R\right) \rightarrow \operatorname{Ext}_{R}^{g-1}\left(B_{r-g}, R\right) \rightarrow \operatorname{Ext}_{R}^{g}\left(\omega_{R / I}, R\right) \rightarrow \operatorname{Ext}_{R}^{g}\left(Z_{r-g}, R\right) \rightarrow 0 .
$$


To determine all of the $R$-modules $\operatorname{Ext}_{R}^{i}\left(B_{r-g}, R\right)$, consider the following exact complex, which is a truncation of the Koszul complex $K_{\bullet}(f ; R)$ :

$$
\mathcal{T}_{\bullet}: 0 \rightarrow K_{r} \rightarrow \cdots \rightarrow K_{r-g+1} \rightarrow B_{r-g} \rightarrow 0 .
$$

Let $\mathcal{I}^{\bullet}$ be an injective resolution of $R$. The double complex $\operatorname{Hom}_{R}\left(\mathcal{T}_{\bullet}, \mathcal{I}^{\bullet}\right)$ whose $(-i)$-th column is $\operatorname{Hom}_{R}\left(\mathcal{T}_{r-g+i}, \mathcal{I}^{j}\right)$ for all $j \geq 0$ gives rise to two spectral sequences where ${ }^{1} E_{\text {hor }}={ }^{\infty} E_{\text {hor }}=0$ and

$$
{ }^{2} E_{\text {ver }}^{-i,-j}=\left\{\begin{array}{cc}
\operatorname{Ext}_{R}^{j}\left(B_{r-g}, R\right), & i=0 \text { and } j \geq 1 \\
0, & i \geq 1 \text { and } j \geq 1, \\
H_{g-i}(f ; R,) & i \geq 2 \text { and } j=0 .
\end{array}\right.
$$

Notice that the only non-trivial map arising from this spectral sequence which is living in ${ }^{i} E_{v e r}$ for $2 \leq i \leq g+1$ is ${ }^{i} \mathrm{~d}_{\text {ver }}^{0,-i+1}$ : $\operatorname{Ext}_{R}^{i-1}\left(B_{r-g}, R\right) \rightarrow H_{g-i}(f ; R)$. Therefore as ${ }^{\infty} E_{\text {ver }}=0$, all these maps must be isomorphisms, which proves (iv). Also, if $g \geq 2$ it shows that $\operatorname{Ext}_{R}^{g-1}\left(B_{r-g}, R\right) \cong R / I$.

We now separate the cases $g=1$ and $g \geq 2$. First let $g \geq 2$. Notice that $\operatorname{Ext}_{R}^{g}\left(\omega_{R / I}, R\right) \cong \operatorname{End}_{R}\left(\omega_{R / I}\right)$. Then, by modifying the maps in (2.1), we have the following exact sequence:

$$
0 \rightarrow \operatorname{Ext}_{R}^{g-1}\left(Z_{r-g}, R\right) \rightarrow R / I \stackrel{\eta}{\rightarrow} \operatorname{End}_{R}\left(\omega_{R / I}\right) \rightarrow \operatorname{Ext}_{R}^{g}\left(Z_{r-g}, R\right) \rightarrow 0,
$$

where $\eta$ is given by multiplication by $\eta(1)$. Now let $\mathfrak{p} \supseteq I$ be a prime ideal of height at most $g+1$. Then $\operatorname{Ext}_{R}^{g}\left(Z_{r-g}, R\right)_{\mathfrak{p}}=0$ (by Remark 2.4(iii)), which implies that $\eta(1)$ is a unit in $\operatorname{End}_{R}\left(\omega_{R / I}\right)_{\mathfrak{p}}$. The Krull principal ideal theorem applied to the ring $\operatorname{End}_{R}\left(\omega_{R / I}\right)$ then implies that $\eta(1)$ is a unit in $\operatorname{End}_{R}\left(\omega_{R / I}\right)$. Therefore $\operatorname{Ext}_{R}^{g}\left(Z_{r-g}, R\right) \cong \operatorname{Coker} \eta \cong \operatorname{Coker}\left(R / I \stackrel{\text { can. }}{\longrightarrow} \operatorname{End}_{R}\left(\omega_{R / I}\right)\right)$, which yields (ii) for $g \geq 2$.

For (iii), recall that $\eta$ induces a homomorphism $\bar{\eta}: R / I^{u n m} \rightarrow \operatorname{End}_{R}\left(\omega_{R / I}\right)$ with Coker $\bar{\eta} \cong$ Coker $\eta$. As mentioned above, $\bar{\eta}_{\mathfrak{p}}$ is onto for all $\mathfrak{p} \supseteq I$ with ht $(\mathfrak{p})=g$. On the other hand, for such a prime ideal $\operatorname{End}\left(\omega_{R / I}\right)_{\mathfrak{p}} \cong(R / I)_{\mathfrak{p}} \cong\left(R / I^{\text {unm }}\right)_{\mathfrak{p}}$. Hence the composed map from $(R / I)_{\mathfrak{p}}$ to itself is an isomorphism which implies that $(\bar{\eta})_{\mathfrak{p}}$ is an isomorphism, so that $\bar{\eta}$ is injective, since $\operatorname{Ass}(\operatorname{Ker} \bar{\eta}) \subseteq \operatorname{Ass}\left(R / I^{u n m}\right)$. Now (iii) follows from the commutative diagram

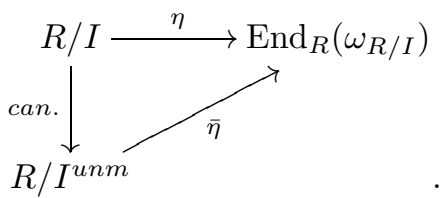

We now turn to the case where $g=1$. Note that in this case $B_{r-g} \cong R$; thus the exact sequence (2.1) can be written as

$$
0 \rightarrow \operatorname{Hom}_{R}\left(Z_{r-1}, R\right) \rightarrow R \stackrel{\eta}{\rightarrow} \operatorname{End}_{R}\left(\omega_{R / I}\right) \rightarrow \operatorname{Ext}_{R}^{1}\left(Z_{r-1}, R\right) \rightarrow 0 .
$$

One then shows, as above, that $\eta(1)$ is a unit in $\operatorname{End}_{R}\left(\omega_{R / I}\right)$ and $\operatorname{Ker} \eta=$ $\operatorname{Ker}\left(R / I \stackrel{\text { can. }}{\longrightarrow} \operatorname{End}_{R}\left(\omega_{R / I}\right)\right)=\operatorname{Ann}\left(\operatorname{End}_{R}\left(\omega_{R / I}\right)\right)=I^{u n m}$. Hence, by local duality, $H_{\mathfrak{m}}^{d}\left(Z_{r-1}\right) \cong\left(I^{u n m}\right)^{v}$. This proves $(\mathrm{v})$.

Replacing Ker $\eta$ by $I^{u n m}$ in (2.3), we have the following short exact sequence which immediately completes the proof of (ii) for the case $g=1$ :

$$
0 \rightarrow R / I^{u n m} \stackrel{\bar{n}}{\rightarrow} \operatorname{End}_{R}\left(\omega_{R / I}\right) \rightarrow \operatorname{Ext}_{R}^{1}\left(Z_{r-1}, R\right) \rightarrow 0 .
$$


By Remark 2.4(iii), $\operatorname{Ext}_{R}^{1}\left(Z_{r-1}, R\right)_{\mathfrak{p}}=0$ for all $\mathfrak{p} \supseteq I$ with ht $(\mathfrak{p})=1,2$. Therefore $\operatorname{dim}\left(\operatorname{Ext}_{R}^{1}\left(Z_{r-1}, R\right)\right) \leq \operatorname{dim}(R / I)-2$. Now if $R / I^{\text {unm }}$ is $\mathrm{CM}$ or even satisfies $S_{2}$, then both $R / I^{u n m}$ and $\operatorname{End}_{R}\left(\omega_{R / I}\right)$ satisfy the Serre condition $\mathrm{S}_{2}$ so that $\operatorname{depth}\left(\operatorname{Ext}_{R}^{1}\left(Z_{r-1}, R\right)_{\mathfrak{p}}\right) \geq 1$ for the same prime ideals $\mathfrak{p}$, which implies that $\operatorname{Ext}_{R}^{1}\left(Z_{r-1}, R\right)=0$. Now (1) follows from this fact and (v), while (2) and (3) are immediate consequences of (i)-(iv). We just mention that if $\mathfrak{b}$ is an ideal in the Gorenstein ring $R$, then $\omega_{R / \mathfrak{b}}$ is $\mathrm{CM}$ and $R / \mathfrak{b}$ satisfies $\mathrm{S}_{2}$ if and only if $R / \mathfrak{b}^{\text {unm }}$ is CM.

We return to the complex $\mathcal{Z}_{\bullet}^{+}$to investigate the acyclicity of this complex. In the next theorem it is shown that the complex $\mathcal{Z}_{\bullet}^{+}$is acyclic for a wide class of ideals.

Theorem 2.7. Suppose that $R$ is a $C M$ local ring and that $J$ is an s-residual intersection of I. If I satisfies $\mathrm{SDC}_{0}$ at level $\min \{s-g-3, r-g\}$, then $\mathcal{Z}_{\bullet}^{+}$is acyclic.

Proof. Invoking the "lemme d'acyclicité" [26] or [3, 1.4.24], we have to show that

(i) $\mathcal{Z}_{\bullet}^{+}$is acyclic on the punctured spectrum and

(ii) $\operatorname{depth}\left(\mathcal{Z}_{i}^{+}\right) \geq i$ for all $i \geq 0$.

(ii) is automatically satisfied due to the condition $\mathrm{SDC}_{0}$. We just recall that $\mathcal{Z}_{i}^{+}=$ $Z_{r-s+i}^{+}$and that Remark 2.4(iii) assures that the mentioned level in the theorem is enough.

We prove (i). Let $\mathfrak{p}$ be a non-maximal prime ideal of $R$. Using induction on $\mathrm{ht}(\mathfrak{p})$, we prove that $\left(\mathcal{Z}_{\bullet}^{+}\right)_{\mathfrak{p}}$ is acyclic. If $\mathrm{ht}(\mathfrak{p}) \leq s-1$, then, by definition of $s$ residual intersections, $\mathfrak{a} R_{\mathfrak{p}}=I R_{\mathfrak{p}}$ which, in conjunction with Lemma 2.2. implies that $\left(\mathcal{Z}_{\bullet}^{+}\right)_{\mathfrak{p}}$ is acyclic. Now assume that $h t(\mathfrak{p}) \geq s$ and that $\left(\mathcal{Z}_{\bullet}^{+}\right)_{\mathfrak{q}}$ is acyclic for any prime ideal $\mathfrak{q}$ with ht $\mathfrak{q}<\operatorname{ht}(\mathfrak{p})$. At this moment we apply the acyclicity's lemma to the complex $\left(\mathcal{Z}_{\bullet}^{+}\right)_{\mathfrak{p}}$. Condition (i) is satisfied by induction hypothesis. To verify condition (ii) for this complex, we consider two cases:

Case 1: $s-3 \leq r$. In this case, by Remark 2.4(iii), $\operatorname{depth}\left(\left(\mathcal{Z}_{i}^{+}\right)_{\mathfrak{p}}\right) \geq 2$ for $i=$ $0,1,2$ (the case where $\operatorname{depth}(R)=1=s$ is trivial). Let $i \geq 3$. Then recalling the level mentioned in the theorem, we have $\operatorname{depth}\left(\left(\mathcal{Z}_{i}^{+}\right)_{\mathfrak{p}}\right)=\operatorname{depth}\left(\left(Z_{r-s+i}^{+}\right)_{\mathfrak{p}}\right)=$ $\min \left\{\operatorname{depth}\left(\left(Z_{r-s+j}\right)_{\mathfrak{p}}\right): j \geq i\right\} \geq \operatorname{ht}(\mathfrak{p})-r+(r-s+i)=\operatorname{ht}(\mathfrak{p})-s+i \geq i$.

Case 2: $s-3 \geq r$. In this case, $\left(\mathcal{Z}_{i}^{+}\right)_{\mathfrak{p}}=\left(Z_{0}\right)_{\mathfrak{p}} \oplus\left(\bigoplus_{j \geq 1}\left(Z_{j}^{e_{i j}}\right)_{\mathfrak{p}}\right)$ for all $0 \leq i \leq s-r$ and some $e_{i j}$. Hence we have to show that $\operatorname{depth}\left(\left(Z_{i}^{+}\right)_{\mathfrak{p}}\right) \geq s-r+i$ for all $i \geq 0$. Remark 2.4(i) implies that $\operatorname{depth}\left(\left(Z_{i}^{+}\right)_{\mathfrak{p}}\right) \geq \operatorname{ht}(\mathfrak{p})-r+i$, where $\operatorname{ht}(\mathfrak{p})-r+i=$ $\operatorname{ht}(\mathfrak{p})-s+s-r+i \geq s-r+i$, as desired.

Now we identify the module Coker $\varphi_{0}$ in $\mathcal{Z}_{\bullet}^{+}$.

Consider the two spectral sequences arising from the double complex $\mathcal{G}:=$ $\mathcal{C}_{\mathfrak{g}}^{\bullet}(S) \otimes_{S} \mathcal{D}$. (see the proof of Lemma 2.2):

$$
\begin{gathered}
\left({ }^{2} E_{\text {hor }}^{-i,-j}\right)_{0}=H_{\mathfrak{g}}^{j}\left(H_{i}\left(\mathcal{D}_{\bullet}\right)\right)_{0} \quad \text { for all } i \text { and } j,\left({ }^{1} E_{\text {ver }}\right)_{0}=\mathcal{Z}_{\bullet}^{+} \text {and } \\
\left({ }^{2} E_{\text {ver }}^{-i,-j}\right)_{0}=\left\{\begin{array}{cl}
H_{i-r}\left(\mathcal{Z}_{\bullet}^{+}\right) & \text {if } j=r \\
0 & \text { otherwise. }
\end{array}\right.
\end{gathered}
$$

Recall that the degree of a homomorphism in ${ }^{i} E_{\text {hor }}$ is $(-i+1,-i)$. Thus $\left({ }^{\infty} E_{h o r}^{0,0}\right)_{0} \subset$ $\left({ }^{2} E_{\text {hor }}^{0,0}\right)_{0}=H_{\mathfrak{g}}^{0}\left(H_{0}\left(\mathcal{D}_{\bullet}\right)\right)_{0} \subseteq R$. On the other hand, by the convergence of $\left({ }^{2} E_{\text {hor }}^{-i,-j}\right)_{0}$ to the homology modules of $\mathcal{Z}_{\bullet}^{+}$, there exists a filtration of $H_{0}\left(\mathcal{Z}_{\bullet}^{+}\right)=$Coker $\varphi_{0}$, 
say $\cdots \subseteq \mathcal{F}_{2} \subseteq \mathcal{F}_{1} \subseteq \operatorname{Coker} \varphi_{0}$, such that Coker $\varphi_{0} / \mathcal{F}_{1} \cong\left({ }^{\infty} E_{\text {hor }}^{0,0}\right)_{0}$. Therefore defining $\tau$ as the composition of the homomorphisms

$$
Z_{r-s}^{+} \stackrel{\text { can. }}{\longrightarrow} \text { Coker } \varphi_{0} \stackrel{\text { can. }}{\longrightarrow} \text { Coker } \varphi_{0} / \mathcal{F}_{1} \cong\left({ }^{\infty} E_{h o r}^{0,0}\right)_{0} \subseteq R,
$$

we have another complex of $R$-modules $\mathcal{C}_{\bullet}:=\mathcal{Z}_{\bullet}^{+} \stackrel{\tau}{\rightarrow} R \rightarrow 0$.

Proposition 2.8. Suppose that $R$ is a $C M$ local ring and that $J$ is an s-residual intersection of $I$. If I satisfies $\mathrm{SDC}_{1}$ at level $\min \{s-g-2, r-g\}$, then $\mathcal{C} \bullet$ is acyclic.

Proof. The proof will be in the same way as the proof of Theorem 2.7. Notice that the identification Coker $\varphi_{0} \cong R$ in Lemma 2.2 is given by $\tau$.

As an application of mentioning the levels in Theorem 2.7 and Proposition 2.8, one sees that in the case where the residual intersection is close to the linkage, the acyclicity of $\mathcal{Z}_{\bullet}^{+}$and $\mathcal{C}_{\bullet}$ follow automatically, without any extra assumption on $I$.

Corollary 2.9. If $R$ is a $C M$ local ring and $J$ is an s-residual intersection of $I$, then

(a) $\mathcal{Z}_{\bullet}^{+}$is acyclic if one of the following conditions holds:

(i) $s \leq g+2$ or

(ii) $s=g+3$ and $H_{r-g}(f ; R)$ is $C M$.

(b) $\mathcal{C}_{\bullet}$ is acyclic if one of the following conditions holds:

(i) $s \leq g+1$ or

(ii) $s=g+2, R$ is Gorenstein and $I^{u n m}$ is $C M$.

Proof. All parts are immediate consequences of Theorem 2.7 and Proposition 2.8 , Both (a)(i) and (b)(i) follow from the fact that $\mathrm{SDC}_{1}$ at level -1 is always satisfied by Remark 2.4(iii). Under the condition of (a)(ii), one sees that $I$ satisfies $\mathrm{SDC}_{0}$ at level 0 by Remark 2.4(iv). Also (b)(ii) is implied by Remark 2.4(v) since $I$ satisfied $\mathrm{SDC}_{1}$ at level 0 .

Example 2.10. C. Huneke, in [18, 3.3], provides an example of a CM ideal $I$ in a regular local ring with a 4-residual intersection which is not CM. In this example $r=6, s=4$, and $g=3$. Hence Corollary 2.9(b)(i) shows that the complex $\mathcal{C}_{\bullet}$, associated to the ideals in [18, 3.3], is acyclic. Also, it will be seen from Theorem 2.11 that the ideal $I$ in this example is an example of a CM ideal in a regular local ring which satisfies $G_{\infty}$, generated by a proper sequence [14, $5.5\left(i v_{a}\right)$ and $12.9(2)$ ] but doesn't satisfy $\mathrm{SDC}_{1}$ at level +1 .

We are now ready to establish the first main theorem in this paper.

Theorem 2.11. Suppose that $(R, \mathfrak{m})$ is a $C M{ }^{*}$ local ring and that $J=\mathfrak{a}: I$ is an $s$-residual intersection of $I$, where $I$ and $\mathfrak{a}$ are homogeneous ideals. If $I$ satisfies $\mathrm{SDC}_{1}$ at level $\min \{s-g, r-g\}$, then either $J=R$ or there exists a homogeneous ideal $K \subseteq J$ such that

(i) $K$ is $C M$ of height $s$;

(ii) $\mathrm{V}(K)=\mathrm{V}(J)$;

(iii) $K=J$ off $\mathrm{V}(I)$;

(iv) $K=J$, whenever $I / \mathfrak{a}$ is generated by at most one element locally in height s. In this case $R / J$ is resolved by the complex $\mathcal{C}$. associated to $I$ and $\mathfrak{a}$. 
Proof. The fact that every ideal we consider is homogeneous enables us to pass to the local ring $R_{\mathfrak{m}}$. Henceforth we assume $(R, \mathfrak{m})$ is a CM local ring.

Consider the complex $\mathcal{C}$. associated to $I$ and $\mathfrak{a}$. We prove that the ideal $K:=$ $\operatorname{Im} \tau$ satisfies the desired properties. The convergence of the spectral sequences arising from $\mathcal{G}:=\mathcal{C}_{\mathfrak{g}}^{\bullet}(S) \otimes_{S} \mathcal{D}_{\bullet}$, in conjunction with the fact that $H_{\mathfrak{g}}^{r}\left(D_{r}\right)_{1}=0$ (see the paragraph preceding Lemma 2.1), implies that $\left({ }^{\infty} E_{\text {hor }}^{-i,-i}\right)_{1}=0$ for all $i \geq 0$. (Further, one can see that $\left({ }^{\infty} E_{\text {hor }}^{0,0}\right)_{j}=0$ for $j \geq 1$; thus $\operatorname{Im} \tau={ }^{\infty} E_{\text {hor }}^{0,0}=$ $\left({ }^{\infty} E_{\text {hor }}^{0,0}\right)_{0}$. $)$ In particular, $\mathfrak{g}(\operatorname{Im} \tau) \subseteq\left({ }^{\infty} E_{\text {hor }}^{0,0}\right)_{1}=0$. That is, $\operatorname{Im} \tau \subseteq J$.

If $J=R$, then Lemma 2.2 implies that $\operatorname{Im}(\tau)=R$. Henceforth to avoid the trivial cases, we assume that neither $J$ nor $\operatorname{Im}(\tau)$ is the unit ideal.

Notice that by Proposition 2.8 the $\mathrm{SDC}_{1}$ condition on $I$ implies that the complex $\mathcal{C}_{\bullet}$ is acyclic.

To prove (i), recall that for any prime $\mathfrak{p}$ with $h t(\mathfrak{p}) \leq s-1, \mathfrak{a} R_{\mathfrak{p}}=I R_{\mathfrak{p}}$; hence by Lemma 2.2. $\left(\mathcal{C}_{\bullet}\right)_{\mathfrak{p}} \rightarrow 0$ is exact. That is, $(\operatorname{Im} \tau)_{\mathfrak{p}}=R_{\mathfrak{p}}$. Thus $\mathfrak{p}$ does not contain $\operatorname{Im} \tau$. Therefore $\operatorname{ht}(\operatorname{Im} \tau) \geq s$. On the other hand, considering the double complex $\mathcal{C}_{\mathfrak{m}}(R) \otimes_{R} \mathcal{C}_{\bullet}$, the condition $\operatorname{SDC}_{1}$ on $I$ implies that $\operatorname{depth}(R / \operatorname{Im} \tau)=$ $\operatorname{depth}\left(H_{0}\left(\mathcal{C}_{\bullet}\right)\right) \geq d-s$. Therefore $R / \operatorname{Im} \tau$ is $\mathrm{CM}$ of dimension $d-s$.

For (ii) it is enough to show that $\mathrm{V}(\operatorname{Im} \tau) \subseteq \mathrm{V}(J)$. Let $\mathfrak{p}$ be a prime ideal not containing $J$; then $\mathfrak{a} R_{\mathfrak{p}}=I R_{\mathfrak{p}}$. Then Lemma 2.2 implies that $(\operatorname{Im} \tau)_{\mathfrak{p}}=R_{\mathfrak{p}}$, and this completes the proof.

(iii) is a special case of (iv). For (iv), since $\operatorname{Im} \tau \subseteq J$ and $\operatorname{Im} \tau$ is $\mathrm{CM}$ of height $s$ by (i), it is enough to show that $\operatorname{Im} \tau$ and $J$ coincide locally in height $s$. Let $\mathfrak{p}$ be a prime ideal of height $s$. We may (and do) replace $R$ by $R_{\mathfrak{p}}$ and assume that $\mu(I / \mathfrak{a}) \leq 1$. It follows that $\mathfrak{g} \mathcal{S}_{I}=(\gamma) \mathcal{S}_{I}+x \mathcal{S}_{I}$ for some $x \in\left(\mathcal{S}_{I}\right)_{1}$. Since $\operatorname{Supp}\left(H_{i}\left(\mathcal{D}_{\bullet}\right)\right) \subseteq \mathrm{V}\left((\gamma) \mathcal{S}_{I}\right)$ for all $i$ (see Lemma 2.2 and its proof), $H_{\mathfrak{g}}^{j}\left(H_{i}\left(\mathcal{D}_{\bullet}\right)\right) \cong$ $H_{(x)}^{j}\left(H_{i}\left(\mathcal{D}_{\bullet}\right)\right)$ for all $j$. Thus $H_{\mathfrak{g}}^{j}\left(H_{i}\left(\mathcal{D}_{\bullet}\right)\right)=0$ for all $j \geq 2$. It then follows that $H_{\mathfrak{g}}^{0}\left(\mathcal{S}_{I} /(\gamma) \mathcal{S}_{I}\right)={ }^{2} E_{\text {hor }}^{0,0}={ }^{\infty} E_{\text {hor }}^{0,0}$.

Now the result follows from the inclusion

$$
{ }^{\infty} E_{\text {hor }}^{0,0}=\operatorname{Im} \tau \subseteq J \subseteq H_{\mathfrak{g}}^{0}\left(\mathcal{S}_{I} /(\gamma) \mathcal{S}_{I}\right)_{0} .
$$

The condition imposed on Theorem 2.11(iv) is not as restricting. Indeed this condition replaces with the conditions $G_{s}$ and the geometric residual intersection in the literatures; e.g., [7, 18, 16, 19. Theorem 2.11(iv) is a good progress in affirmatively answering one of the main open questions in the theory of residual intersections [19, Question 5.7]. As a corollary one can give a positive answer for this question in the geometric case.

Corollary 2.12. Suppose that $R$ is a CM local ring and I satisfies the sliding depth condition, SD. Then any geometric residual intersection of I is CM.

Despite the complexity of the structure of the ideal $K$ introduced in Theorem 2.11, it is shown in the next proposition that under some conditions this ideal is a specialization of the generic one.

We first recall the circumstances of the generic case. Besides the notation at the beginning of the section, assume that $(R, \mathfrak{m})$ is a Noetherian local ring and that $l_{i}=\sum_{j=1}^{r} c_{i j} f_{j}$ for all $i=1, \cdots, s$. Let $U=\left(U_{i j}\right)$ be a generic $s$ by $r$ matrix, $\tilde{R}=R[U]_{\left(\mathfrak{m}, U_{i j}-c_{i j}\right)}, \tilde{S}=\tilde{R}\left[T_{1}, \cdots, T_{r}\right], \tilde{l}_{i}=\sum_{j=1}^{r} U_{i j} f_{j}, \tilde{\gamma}_{i}=\sum_{j=1}^{r} U_{i j} T_{j}$ for all $i=1, \cdots, s, \tilde{\gamma}=\left(\tilde{\gamma}_{1}, \cdots, \tilde{\gamma}_{s}\right), \tilde{\mathfrak{a}}=\left(\tilde{l}_{1}, \cdots, \tilde{l}_{s}\right)$ and $\tilde{J}=\tilde{\mathfrak{a}}: \tilde{R} I \tilde{S}$. Consider the 
standard grading of $\tilde{S}=\tilde{R}\left[T_{1}, \cdots, T_{r}\right]$ by setting $\operatorname{deg}\left(T_{i}\right)=1$. Now replacing the base ring $R$ by the ring $\tilde{R}$, we can construct the double complex $\tilde{\mathcal{E}}:=\tilde{\mathcal{Z}}_{\bullet}^{\prime} \otimes_{\tilde{S}} K_{\bullet}(\tilde{\gamma} ; \tilde{S})$. Consequently, $\tilde{D}_{i}:=D_{i} \otimes_{S} \tilde{S}$. It then follows from the construction of the complex $\mathcal{Z}_{\bullet}^{+}$that

$$
\begin{aligned}
\tilde{\mathcal{Z}}_{i}^{+} & =\left(H_{\mathfrak{g}}^{r}(\tilde{S}) \otimes_{\tilde{S}} \tilde{D}_{i}\right)_{0} \cong\left(\left(H_{\mathfrak{g}}^{r}(S) \otimes_{S} \tilde{S}\right) \otimes_{\tilde{S}}\left(D_{i} \otimes_{S} \tilde{S}\right)\right)_{0} \\
& \cong\left(\left(H_{\mathfrak{g}}^{r}(S) \otimes_{S} D_{i}\right) \otimes_{S} S[U]\right)_{0} \cong\left(H_{\mathfrak{g}}^{r}(S) \otimes_{S} D_{i}\right)_{0} \otimes_{R} R[U]_{\left(\mathfrak{m}, U_{i j}-c_{i j}\right)} \\
& =\mathcal{Z}_{i}^{+}[U]_{\left(\mathfrak{m}, U_{i j}-c_{i j}\right)}
\end{aligned}
$$

Before proceeding, we recall the definition of the deformation in the sense of 20 , Definition 2.1]. Let $(R, \mathfrak{b})$ and $(\tilde{R}, \tilde{\mathfrak{b}})$ be pairs of Noetherian local rings with ideals $\mathfrak{b} \subseteq R$ and $\tilde{\mathfrak{b}} \subseteq \tilde{R}$. We say that $(\tilde{R}, \tilde{\mathfrak{b}})$ is a deformation of $(R, \mathfrak{b})$ if there exists a sequence $\alpha \subseteq \tilde{R}$ which is regular on both $\tilde{R}$ and $\tilde{R} / \tilde{\mathfrak{b}}$ such that $\tilde{R} / \alpha \cong R$ and $(\tilde{\mathfrak{b}}+\alpha) / \alpha \cong \mathfrak{b}$.

Proposition 2.13. With the notation introduced above, if I satisfies the $S D C_{1}$ condition at level $\min \{s-g-2, r-g\}$ and if $J$ and $\tilde{J}$ are s-residual intersections of $I$ and $\tilde{I}$, respectively, then $(\tilde{R}, \tilde{K})$ is a deformation of $(R, K)$, via the sequence $\left(U_{i j}-c_{i j}\right)$.

Proof. The hypotheses of the proposition, in conjunction with Proposition 2.8, imply that both $\mathcal{C}_{\bullet}$ and $\tilde{\mathcal{C}}_{\bullet}$ are acyclic. In the case where $\mathcal{C} \bullet$ is acyclic, the commutative diagram

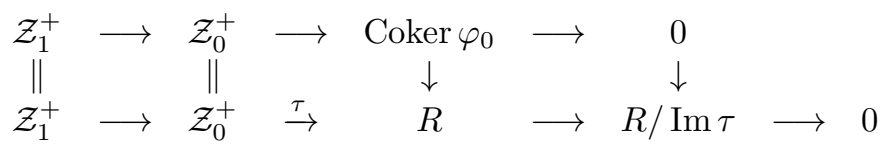

shows that the map Coker $\varphi_{0} \rightarrow \operatorname{Im} \tau$ induced by this diagram is injective. Therefore considering the canonical homomorphisms in (2.4) defining $\tau, \mathcal{F}_{1}=0$. This fact implies that $\left({ }^{\infty} E_{\text {hor }}^{-i,-i}\right)_{0}=0$ for all $i \geq 1$.

Hence

$$
K:=\operatorname{Coker} \varphi_{0}=\operatorname{Im} \tau\left(={ }^{\infty} E_{\text {hor }}^{0,0}=H_{\mathfrak{g}}^{0}\left(\mathcal{S}_{I} /(\gamma) \mathcal{S}_{I}\right)_{0}\right) .
$$

Similarly the acyclicity of $\tilde{\mathcal{C}}_{\bullet}$ implies that $\tilde{K}:=\operatorname{Im}(\tilde{\tau})=\operatorname{Coker}\left(\tilde{\varphi}_{0}\right)$.

Let $\pi$ be the epimorphism of $\tilde{R}$ to $R$ sending $U_{i j}$ to $c_{i j}$. One has $\pi\left(K_{\bullet}(\tilde{\gamma} ; \tilde{S})\right)=$ $K_{\bullet}(\gamma ; S)$ so that $\pi\left(\tilde{\mathcal{D}}_{\bullet}\right)=\mathcal{D}_{\bullet}$, and then (2.5) shows that $\pi\left(\tilde{\mathcal{Z}}_{\bullet}^{+}\right)=\mathcal{Z}_{\bullet}^{+}$. This in turn implies that $\pi(\tilde{K})=K$.

Clearly, the sequence $\left(U_{i j}-c_{i j}\right)$ is a regular sequence on $\tilde{R}$. Thus to prove that $(\tilde{R}, \tilde{K})$ is a deformation of $(R, K)$ it just remains to prove that $\left(U_{i j}-c_{i j}\right)$ is a regular sequence on $\tilde{R} / \tilde{K}$. To this end, consider the double complex $K_{\bullet}\left(U_{i j}-c_{i j} ; \tilde{R}\right) \otimes_{\tilde{R}} \tilde{\mathcal{C}}$. In view of (2.5),$\left(U_{i j}-c_{i j}\right)$ is a regular sequence on $\tilde{\mathcal{C}}_{i}$ for all $i$. Therefore the first terms in the vertical spectral sequence arising from this double complex has the form ${ }^{1} E_{\text {ver }}^{-p, 0} \cong \mathcal{C}_{p}$ and ${ }^{1} E_{\text {ver }}^{-p,-q}=0$ whenever $q \neq 0$, and ${ }^{2} E_{\text {ver }}^{-p, 0} \cong H_{p}\left(\mathcal{C}_{\bullet}\right)$. On the other hand, since $\tilde{\mathcal{C}}_{\bullet}$ is acyclic, ${ }^{1} E_{\text {hor }}^{0,-q}=K_{q}\left(U_{i j}-c_{i j} ; \tilde{R} / \tilde{K}\right)$ and ${ }^{1} E_{\text {hor }}^{-p,-q}=0$ if $p \neq 0$, and ${ }^{2} E_{\text {hor }}^{0,-q}=H_{q}\left(U_{i j}-c_{i j} ; \tilde{R} / \tilde{K}\right)$ for all $q$. Hence both spectral sequences abut at the second step, and this provides an isomorphism $H_{t}\left(\mathcal{C}_{\bullet}\right) \cong H_{t}\left(U_{i j}-c_{i j} ; \tilde{R} / \tilde{K}\right)$ for all $t$. Now the result follows from the acyclicity of $\mathcal{C}_{\bullet}$. 
In the issues concerning the residual intersection, there are some slightly weaker conditions than the $G_{s}$ condition. One of these conditions which we call the $G_{s}^{-}$ condition first appeared in 14 to prove the acyclicity of the $\mathcal{Z}$-complex. Similar to the definition of the $G_{s}$ condition, we say that an ideal $I$ satisfies the $G_{s}^{-}$condition if $\mu\left(I_{\mathfrak{p}}\right) \leq \mathrm{ht}(\mathfrak{p})+1$ for all $\mathfrak{p} \supseteq I$ with $\mathrm{ht}(\mathfrak{p}) \leq s-1$. While the $G_{s}$ condition is equivalent to existence of geometric $i$-residual intersections for all $i \leq s-1$, the $G_{s}^{-}$condition is equivalent to the existence of (not necessarily geometric) $i$-residual intersections for all $i \leq s-1$. The next proposition is an extension of [5, 3.8].

Proposition 2.14. With the notation and assumptions as in Proposition 2.13, if in addition $I$ satisfies the $G_{s+1}^{-}$condition, then $K=\pi(\tilde{J})$.

Proof. Once we show that the $G_{s+1}^{-}$condition of $I$ implies that $\mu(I \tilde{S} / \tilde{\mathfrak{a}}) \leq 1$ locally in height $s$, this remark is an immediate consequence of Theorem 2.11)(iv) and Proposition 2.13. We avail ourselves of the proof of [20, Lemma 3.1] to show $\mu(I \tilde{S} / \tilde{\mathfrak{a}}) \leq 1$.

Let $Q$ be a prime ideal of $\operatorname{Spec}(\tilde{S})$ with $h t(Q) \leq s$ and $\mathfrak{p}=Q \cap \tilde{R}$, and let $t:=\operatorname{ht}(\mathfrak{p}) \leq s$. With the same argument as in the proof of [20, Lemma 3.1], we may assume that $\tilde{I}_{\mathfrak{p}} \tilde{S}_{Q}$ is generated by at most $t+1$ elements in $\tilde{S}_{Q}$ and assume that $U$ is a $(t+1) \times s$ matrix. Therefore the mapping cone of the following diagram whose rows are free resolutions provides a free resolution for $\tilde{I}_{\mathfrak{p}} \tilde{S}_{Q} / \tilde{\mathfrak{a}} \tilde{S}_{Q}$ :

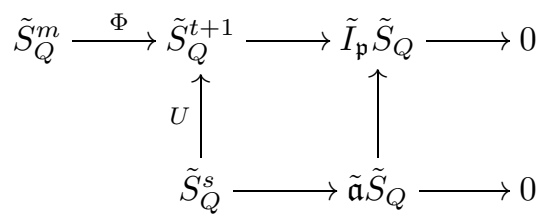

By the Fitting theorem, to prove the assertion it is enough to show that $I_{t}(\Phi \mid U) \nsubseteq Q$, and to this end, it is enough to show that $I_{t}(U) \nsubseteq Q$. If, by contrary, we assume that $I_{t}(U) \subseteq Q$, then $\operatorname{ht}\left(I_{t}(U)_{Q}\right)=(s+1-s+1)(s-t+1)+\operatorname{ht}(\mathfrak{p})=$ $2 s-t+1=s+(s-t)+1 \geq s+1$, which is a contradiction.

As it can be seen from the proof of Theorem 2.11(iv), we use the local condition of generators on $I / \mathfrak{a}$ to show that there exists an element $x$ in $R$ such that $\mathfrak{a}+(x)$ and $I$ have the same radical in $\mathcal{S}_{I}$. Therefore, one may wonder whether to replace the latter condition with that in Theorem 2.11(iv). Now it is natural to ask about the properties of the ideal $\mathfrak{a} \subseteq I$ in $R$ such that $\mathfrak{a} \mathcal{S}_{I}$ has the same radical as $\mathcal{S}_{I+}$. By using the same argument as in the proof of Theorem 2.11(iv), it can be shown that if $\mathfrak{a} \mathcal{S}_{I}$ and $\mathcal{S}_{I+}$ have the same radical, then $\mathfrak{a}=I$.

Equivalently, we see in Proposition 2.16 that the symmetric analogue to the ordinary reduction theory is vacuous. To be more precise, for an ideal $I$ in a commutative ring, we say that the ideal $(\gamma) \subseteq \mathcal{S}_{I}$, generated by elements of degree 1 , is a symmetric reduction of $I$ if $\operatorname{Sym}^{t+1}(I)=(\gamma) \operatorname{Sym}^{t}(I)$ for some integer $t$. Notice that if $I$ is of linear type, this definition and the known definition of reduction coincide.

Here, we provide an elementary proof to Proposition 2.16 which is quite general. Let $A$ be a commutative ring with 1 .

Lemma 2.15. Let $X$ be a set of indeterminates and $B=A[X]$. If $P$ is an ideal generated by linear forms in $B$ whose radical is $(X)$, then $P=(X)$. 
Proof. Suppose $x$ is an element of $X$ and $\left\{p_{i}\right\}$ is a set of linear forms that generate $P$. Let $t$ be an integer such that $x^{t}=\sum_{i=1}^{m} q_{i} p_{i}$ for some integer $m$ and some $q_{i} \in B$. By homogeneity of $x^{t}$ and $p_{i}$, we may assume that each $q_{i}$ is homogeneous of degree $t-1$. Further, if $q_{i}=b_{i} x^{t-1}+q_{i}^{\prime}$ with $\operatorname{deg}_{x} q_{i}^{\prime}<t-1$, we have $x^{t}=\sum_{i=1}^{m} b_{i} x^{t-1} p_{i}$. It then follows that $x \in P$, since $x^{t-1}$ is a non-zero devisor in $B$.

Proposition 2.16. Let I be an ideal in a commutative ring. Then

(i) I has no proper symmetric reduction and

(ii) if I is an ideal of linear type, it has no (ordinary) proper reduction.

Proof. (i) Let $\mathfrak{a} \subseteq I$ be two ideals of a commutative ring $A$ such that $\mathfrak{a} S y m_{A}(I)$ is a symmetric reduction of $I$. $\operatorname{Sym}_{A}(I)=A[X] / \mathcal{L}$, where $X$ is a set of indeterminates and $\mathcal{L}$ is an ideal of linear forms in $A[X]$. If we denote the preimage of $\mathfrak{a} S y m_{A}(I)$ in $A[X]$ by $\mathfrak{a}^{\prime}$, then the assumptions imply that the radical ideal of $\mathfrak{a}^{\prime}+\mathcal{L}$ is $(X)$. Whence the result follows from Lemma 2.15. (ii) is an immediate consequence of (i), since for ideals of linear type, symmetric reductions and ordinary reductions coincide.

Remark 2.17. To the best of our knowledge, the fact that ideals of linear type have no proper reduction is known in the case where $A$ is a Noetherian local ring. Here, the only assumption is "commutative".

\section{Castelnuovo-Mumford Regularity of Residual intersections}

Hereupon our goal is to estimate the regularity of residual intersections of ideals that satisfy some sliding depth conditions. We use three approaches to this end. One is based on the resolution of residual intersections introduced in Theorem 2.11the complex $\mathcal{C}_{\boldsymbol{}}$. The second is based on the structure of the canonical module of the residual intersections-the work of C. Huneke and B. Ulrich in the local case [19, 2.3]. Studying the regularity in the second way provides a criterion to describe when the regularity attains the proposed upper bound. The third approach is the Eagon-Northcott complex to show the equality of the proposed upper bound for perfect ideals of height 2 .

Throughout this section $R=\bigoplus_{n \geq 0} R_{n}$ is a positively graded *local Noetherian ring of dimension $d$ with the graded maximal ideal $\mathfrak{m}$. The maximal ideal of the base ring $R_{0}$ is denoted by $\mathfrak{m}_{0} . I$ and $\mathfrak{a}$ are graded ideals of $R$ generated by homogeneous elements $f_{1}, \cdots, f_{r}$ and $l_{1}, \cdots, l_{s}$, respectively. Let $\operatorname{deg} f_{t}=i_{t}$ for all $1 \leq t \leq r$ with $i_{1} \geq \cdots \geq i_{r}$ and $\operatorname{deg} l_{t}=a_{t}$ for $1 \leq t \leq s$. For a graded ideal $\mathfrak{b}$, the sum of the degrees of a minimal generating set of $\mathfrak{b}$ is denoted by $\sigma(\mathfrak{b})$. Keep other notation as in Section 2. We first recall the definition of the Castelnuovo-Mumford regularity.

Definition 3.1. If $M$ is a finitely generated graded $R$-module, the CastelnuovoMumford regularity of $M$ is defined as $\operatorname{reg}(M):=\max \left\{\operatorname{end}\left(H_{R_{+}}^{i}(M)\right)+i\right\}$.

As an analogue, we define the regularity with respect to the maximal ideal $\mathfrak{m}$ as $\operatorname{reg}_{\mathfrak{m}}(M):=\max \left\{\operatorname{end}\left(H_{\mathfrak{m}}^{i}(M)\right)+i\right\}$.

In the course of the proof of Theorem 3.6 we shall use Proposition 3.4 several times. This proposition has its own interest, as it establishes a relation between $\operatorname{reg}_{\mathfrak{m}}(M)$ and $\operatorname{reg}(M)$. In the proof of Proposition 3.4, we shall use the following two elementary lemmas. 
Lemma 3.2. Suppose that $(A, \mathfrak{m})$ is a Noetherian local ring and denote the Matlis dual $\operatorname{Hom}_{A}\left(-, E_{A}(A / \mathfrak{m})\right)$ by ${ }^{v}$. Let $M$ and $N$ be two $A$-modules such that $\operatorname{dim}\left(N^{v}\right)$ $>\operatorname{dim}\left(M^{v}\right)$. If $\phi: M \rightarrow N$ is an A-homomorphism, then $\operatorname{dim}\left((\operatorname{Coker} \phi)^{v}\right)=$ $\operatorname{dim}\left(N^{v}\right)$.

Lemma 3.3. Suppose that $(A, \mathfrak{m})$ is a Noetherian complete local ring and denote the Matlis dual $\operatorname{Hom}_{A}\left(-, E_{A}(A / \mathfrak{m})\right)$ by ${ }^{v}$. Let $M$ be a finitely generated A-module. Then $\operatorname{dim}\left(H_{\mathfrak{m}}^{i}(M)^{v}\right) \leq i$ for all $i$, and equality holds for $i=\operatorname{dim} M$.

Proposition 3.4. Assume that $R$ is $C M$ and let $M$ be a finitely generated graded $R$-module. Then

$$
\operatorname{reg}(M) \leq \operatorname{reg}_{\mathfrak{m}}(M) \leq \operatorname{reg}(M)+\operatorname{dim}\left(R_{0}\right) .
$$

Proof. Considering the $\mathfrak{m}_{0}$-adic completion of $R_{0}, \widehat{R_{0}}$, we may pass to the CM * complete *local ring $\widehat{R_{0}} \otimes_{R_{0}} R$ via the natural homomorphism $R \rightarrow \widehat{R_{0}} \otimes_{R_{0}} R$ so that in the proof we assume $R$ admits a canonical module; see [2, 15.2.2].

To prove $\operatorname{reg}_{\mathfrak{m}}(M) \leq \operatorname{reg}(M)+\operatorname{dim}\left(R_{0}\right)$, we consider the composed functor spectral sequence $H_{\mathfrak{m}_{0}}^{p}\left(H_{R_{+}}^{q}(M)\right) \Rightarrow H_{\mathfrak{m}}^{p+q}(M)$. Let $i$ be an integer. Notice that for all $p>\operatorname{dim}\left(R_{0}\right), H_{\mathfrak{m}_{0}}^{p}(-)=0$, and also if $\rho>\operatorname{reg}(M)+\operatorname{dim}\left(R_{0}\right)-i$ and $p+q=i$ where $p \leq \operatorname{dim}\left(R_{0}\right)$, then $\rho>\operatorname{end}\left(H_{R_{+}}^{q}(M)\right)+q+\operatorname{dim}\left(R_{0}\right)-i=\operatorname{end}\left(H_{R_{+}}^{q}(M)\right)+$ $\operatorname{dim}\left(R_{0}\right)-p \geq \operatorname{end}\left(H_{R_{+}}^{q}(M)\right)$. Now the result follows from the fact that for any integer $q, H_{R_{+}}^{q}(M)_{\rho}$ is an $R_{0}$-module. Hence $H_{\mathfrak{m}_{0}}^{p}\left(H_{R_{+}}^{q}(M)_{\rho}\right) \cong H_{\mathfrak{m}_{0}}^{p}\left(H_{R_{+}}^{q}(M)\right)_{\rho}$ (cf. [2, 13.1.10]) so that we have the following convergence of the components of the above spectral sequence, $H_{\mathfrak{m}_{0}}^{p}\left(H_{R_{+}}^{q}(M)_{\rho}\right) \Rightarrow H_{\mathfrak{m}}^{p+q}(M)_{\rho}$, which yields the second inequality.

To show that $\operatorname{reg}(M) \leq \operatorname{reg}_{\mathfrak{m}}(M)$, we consider the composed cohomology modules $H_{\mathfrak{m}_{0}}^{p}\left(H_{R_{+}}^{q}(M)\right)$ as second terms of the horizontal spectral sequence arising from the double complex $\mathcal{C}_{\mathfrak{m}_{0}}\left(R_{0}\right) \otimes_{R_{0}} \mathcal{C}_{R_{+}}(M)$. As usual, we put this double complex in the third quadrant in the coordinate plane with $\mathcal{C}_{\mathfrak{m}_{0}}^{0}\left(R_{0}\right) \otimes_{R_{0}} \mathcal{C}_{R_{+}}^{r}(M)$ at the origin, where $r=\max \left\{j: H_{R_{+}}^{j}(M) \neq 0\right\}$, so that ${ }^{2} E_{\text {hor }}^{-p,-q}=H_{\mathfrak{m}_{0}}^{q}\left(H_{R_{+}}^{r-p}(M)\right)$. Now let $i$ be an integer such that $H_{R_{+}}^{i}(M)_{\mu} \neq 0$ for $\mu=\operatorname{reg}(M)-i$.

Let $\delta=\max \left\{j: H_{\mathfrak{m}_{0}}^{j}\left(H_{R_{+}}^{t}(M)_{\mu}\right) \neq 0, j \geq i-t\right.$ for some $\left.t \leq i\right\}$. Notice that $H_{R_{+}}^{i}(M)_{\mu}$ is a finitely generated non-zero $R_{0}$-module and that there exists an integer $j$ such that $H_{\mathfrak{m}_{0}}^{j}\left(H_{R_{+}}^{i}(M)_{\mu}\right) \neq 0$; hence $\delta \geq 0$ (unless it is $-\infty$ ). Let $t \leq i$ be an integer for which $H_{\mathfrak{m}_{0}}^{\delta}\left(H_{R_{+}}^{t}(M)_{\mu}\right) \neq 0$. By definition of $\delta,\left({ }^{2} E_{h o r}^{-(r-p),-q}\right)_{\mu}=0$ for all $p \leq i$ and $q \geq \delta+1$. Thus $\left({ }^{\ell} E_{\text {hor }}^{-(r-t),-\delta}\right)_{\mu}=\operatorname{Coker}\left(\phi_{\ell}\right)$, where

$$
\phi_{\ell}:=\left({ }^{\ell} \mathrm{d}_{\text {hor }}^{-(r-t)+\ell-1,-\delta+\ell}\right)_{\mu}:\left({ }^{\ell} E_{\text {hor }}^{-(r-t)+\ell-1,-\delta+\ell}\right)_{\mu} \rightarrow N_{\ell}:=\left({ }^{\ell} E_{\text {hor }}^{-(r-t),-\delta}\right)_{\mu} .
$$

On the one hand, $\left({ }^{\ell} E_{h o r}^{-(r-t)+\ell-1,-\delta+\ell}\right)_{\mu}^{v}$ is a subquotient of the module

$$
\left({ }^{2} E_{\text {hor }}^{-(r-t)+\ell-1,-\delta+\ell}\right)_{\mu}^{v}=\left(H_{\mathfrak{m}_{0}}^{\delta-\ell}\left(H_{R_{+}}^{t-\ell+1}(M)_{\mu}\right)\right)^{v}
$$

which has dimension at most $\delta-\ell<\delta$ for any $\ell \geq 2$, by Lemma 3.3 .

On the other hand, $N_{2}=H_{\mathfrak{m}_{0}}^{\delta}\left(H_{R_{+}}^{t}(M)_{\mu}\right)$, so that $\left(N_{2}\right)^{v}$ has dimension $\delta$ by Lemma 3.3

Since $N_{\ell+1}=\operatorname{Coker}\left(\phi_{\ell}\right)$, it follows from Lemma 3.2, by recursion on $\ell$, that $\operatorname{dim}\left(\left(N_{\ell}\right)^{v}\right)=\delta$ for all $\ell \geq 2$; in particular, $\left({ }^{\infty} E_{\text {hor }}^{-(r-t),-\delta}\right)_{\mu} \neq 0$. Now the convergence of the spectral sequence, $H_{\mathfrak{m}_{0}}^{p}\left(H_{R_{+}}^{q}(M)\right) \Rightarrow H_{\mathfrak{m}}^{p+q}(M)$, implies that 
$H_{\mathfrak{m}}^{\delta+t}(M)_{\mu} \neq 0$. Therefore $\operatorname{reg}_{\mathfrak{m}}(M) \geq \operatorname{end}\left(H_{\mathfrak{m}}^{\delta+t}(M)\right)+\delta+t \geq \mu+\delta+t \geq \mu+i=$ $\operatorname{reg}(M)$, as desired.

The next proposition follows along the same lines as the proof of Proposition 3.4. Since this proposition is not used in the sequel, we will not detail the required variations. Part (i) of this proposition was already proven in the articles of E. Hyry 21] and N.V. Trung [29].

Proposition 3.5. With the same notation as in Proposition 3.4,

(i) $\max \left\{\operatorname{end}\left(H_{R_{+}}^{i}(M)\right)\right\}=\max \left\{\operatorname{end}\left(H_{\mathfrak{m}}^{i}(M)\right)\right\}$;

(ii) $H_{\mathfrak{m}_{0}}^{p}\left(H_{R_{+}}^{q}(M)\right)=0$ for all integers $p$ and $q$ with $p+q>\operatorname{dim}(M)$.

Everything is now ready to present the second main result in this paper: the regularity of residual intersections.

Theorem 3.6. Suppose that $(R, \mathfrak{m})$ is $C M{ }^{*}$ local, I is a homogeneous ideal which satisfies $\mathrm{SD}_{1}, J=\mathfrak{a}: I$ is an s-residual intersection of $I$, with $\mathfrak{a}$ homogeneous, and $I / \mathfrak{a}$ is generated by at most one element locally in height $s$. Then

$$
\operatorname{reg}(R / J) \leq \operatorname{reg}(R)+\operatorname{dim}\left(R_{0}\right)+\sigma(\mathfrak{a})-(s-g+1) \operatorname{indeg}(I / \mathfrak{a})-s .
$$

Proof. Considering the $\mathfrak{m}_{0}$-adic completion of $R_{0}, \widehat{R_{0}}$, the fact that the natural homomorphism $R \rightarrow \widehat{R_{0}} \otimes_{R_{0}} R$ is faithfully flat enables us to pass to the CM *complete *local ring $\widehat{R_{0}} \otimes_{R_{0}} R$ via this homomorphism so that in the proof we assume that $R$ admits a graded canonical module.

The assumptions of the theorem completely fulfill what is needed for Theorem 2.11(iv). Thus $R / J$ is $\mathrm{CM}$ and is resolved by $\mathcal{C}_{\text {. }}$.

Before continuing, we just notice that in the case where $g=1$, there is no free $R$ module in the tail of $\mathcal{C}_{\bullet}$, that is, $\mathcal{C}_{s}=Z_{r-g}^{+}=Z_{r-1}^{+}$. Nevertheless, the forthcoming proof will be the same for both cases $g=1$ and $g \geq 2$.

We consider the diagram of the double complex $\mathcal{C}_{\mathfrak{m}}^{\bullet}(R) \otimes_{R} \mathcal{C}_{\bullet}$, where $\mathcal{C}_{\mathfrak{m}}(R)$ is the Čech complex with respect to $R$ and $\mathfrak{m}$. As usual we put this double complex in the third quadrant with $\mathcal{C}_{\mathfrak{m}}^{0}(R) \otimes_{R} \mathcal{C}_{0}$ at the origin.

By the acyclicity of $\mathcal{C}_{\bullet}$, we have

$$
{ }^{2} E_{\text {hor }}^{-p,-q}=\left\{\begin{array}{cl}
H_{\mathfrak{m}}^{d-s}(R / J), & p=0 \text { and } q=d-s, \\
0, & \text { otherwise. }
\end{array}\right.
$$

The fact that $\mathcal{C}_{i}$ is free for $i \geq s-g+2$, in conjunction with Proposition 2.5, implies that ${ }^{2} E_{\text {ver }}^{-p,-q}=H_{\mathfrak{m}}^{q}\left(\mathcal{C}_{p}\right)$ is zero if one of the following holds:

- $p=0, q \neq d$.

- $1 \leq p \leq s-g+1$ and $q-p \leq d-s$.

- $p \geq s-g+2$ and $q \neq d$.

It follows that the only non-zero module ${ }^{2} E_{v e r}^{-p,-q}$ with $q-p=d-s$ is ${ }^{2} E_{v e r}^{-s,-d}$. Hence $H_{\mathfrak{m}}^{d-s}(R / J)={ }^{\infty} E_{\text {hor }}^{0,-(d-s)}={ }^{\infty} E_{\text {ver }}^{-s,-d} \subseteq{ }^{2} E_{\text {ver }}^{-s,-d}$ and

$$
\operatorname{end}\left(H_{\mathfrak{m}}^{d-s}(R / J)\right) \leq \operatorname{end}\left({ }^{2} E_{\text {ver }}^{-s,-d}\right) \text {. }
$$

We now have to estimate end $\left({ }^{2} E_{v e r}^{-s,-d}\right)$ to bound the regularity of $R / J$.

In order to estimate end $\left({ }^{2} E_{v e r}^{-s,-d}\right)$, we need to review the construction of the tail of $\mathcal{C}_{\text {. }}$. The ring $S$, introduced in the first section, has a structure as a positively bigraded algebra. Considering $R$ as a subalgebra of $S$, we write the degrees of an element $x$ of $R$ as the 2-tuple $(\operatorname{deg} x, 0)$ with the second entry zero. So, let 
$\operatorname{deg} f_{t}=\left(i_{t}, 0\right)$ for all $1 \leq t \leq r, \operatorname{deg} l_{t}=\left(a_{t}, 0\right)$ for all $1 \leq t \leq s, \operatorname{deg} T_{t}=\left(i_{t}, 1\right)$ for all $1 \leq t \leq s$, and thus $\operatorname{deg} \gamma_{t}=\left(a_{t}, 1\right)$ for all $1 \leq t \leq s$. With this notation the $\mathcal{Z}$-complex has the following shape:

$$
\mathcal{Z}_{\bullet}: 0 \rightarrow Z_{r-1} \otimes_{R} S(0,-r+1) \rightarrow \cdots \rightarrow Z_{1} \otimes_{R} S(0,-1) \rightarrow Z_{0} \otimes_{R} S \rightarrow 0
$$

Consequently,

$$
\mathcal{Z}_{r-1}^{\prime}=R\left(-\sum_{t=1}^{r} i_{t}, 0\right) \otimes_{R}\left(\bigoplus_{i=1}^{g-1} S(0,-r+i)\right)
$$

and, taking into account that $a_{1}, \cdots, a_{s}$ is a minimal generating set of $\mathfrak{a}$,

$$
\begin{aligned}
D_{r+s-1} & =R\left(-\sum_{t=1}^{r} i_{t}, 0\right) \otimes_{R}\left(\bigoplus_{i=1}^{g-1} S(-\sigma(\mathfrak{a}),-s-r+i)\right) \\
& =R\left(-\sum_{t=1}^{r} i_{t}-\sigma(\mathfrak{a}), 0\right) \otimes_{R}\left(\bigoplus_{i=1}^{g-1} S(0,-s-r+i)\right) .
\end{aligned}
$$

By definition of $\mathcal{C}_{\bullet}, \mathcal{C}_{i}=H_{\mathfrak{g}}^{r}\left(D_{r+i-1}\right)_{(*, 0)}$, where by degree $(*, 0)$ we mean degree zero in the second entry and anything in the first entry; in other words degree zero in $S$ with its natural grading. Hence, as in the proof of Proposition 3.4, it follows from the composed functor spectral sequence that $H_{\mathfrak{m}}^{d}\left(H_{\mathfrak{g}}^{r}\left(D_{r+i-1}\right)_{(*, 0)}\right) \cong$ $H_{\mathfrak{g}+\mathfrak{m}}^{r+d}\left(D_{r+i-1}\right)_{(*, 0)}$.

Thus,

$$
{ }^{2} E_{\text {ver }}^{-s,-d}=\operatorname{Ker}\left(H_{\mathfrak{g}+\mathfrak{m}}^{r+d}\left(D_{r+s-1}\right) \longrightarrow H_{\mathfrak{g}+\mathfrak{m}}^{r+d}\left(D_{r+s-2}\right)\right)_{(*, 0)} .
$$

Let $\omega_{R}$ be the graded canonical module of $R$; then $\omega_{S}$ exists and is equal to $\omega_{R}\left[T_{1}, \cdots, T_{r}\right]\left(-\sum_{t=1}^{r} i_{t},-r\right)$. If ${ }^{v}$ denotes the Matlis dual, $\operatorname{Hom}_{R_{0}}\left(-, E_{0}(R / \mathfrak{m})\right)$, it then follows from the graded local duality theorem that

$$
{ }^{2} E_{v e r}^{-s,-d}=\left(\operatorname{Coker}\left(\operatorname{Hom}_{S}\left(D_{r+s-2}, \omega_{S}\right) \rightarrow \operatorname{Hom}_{S}\left(D_{r+s-1}, \omega_{S}\right)\right)\right)_{(*, 0)}^{v} .
$$

Therefore

$$
\operatorname{end}\left({ }^{2} E_{v e r}^{-s,-d}\right)=-\operatorname{indeg}\left(\operatorname{Coker}\left(\operatorname{Hom}_{S}\left(D_{r+s-2}, \omega_{S}\right) \rightarrow \operatorname{Hom}_{S}\left(D_{r+s-1}, \omega_{S}\right)\right)_{(*, 0)}\right) .
$$

Now recall that the map $\theta: D_{r+s-1} \rightarrow D_{r+s-2}$, in the tail of the complex $\mathcal{D}_{\bullet}$, is defined by the $2 \times 1$ matrix $\left(\begin{array}{c}\delta_{s}^{\gamma} \otimes \mathcal{Z}^{\prime}{ }_{r-1} \\ \delta^{\prime} \otimes K_{s}(\gamma ; S)\end{array}\right)$, where $\delta_{s}^{\gamma}$ is the last map in the Koszul complex $K_{\bullet}(\gamma ; S)$ and $\delta^{\prime}$ is the most-left map in $\mathcal{Z}^{\prime}$. Thus there exists an epimorphism from $\operatorname{Coker}\left(\operatorname{Hom}_{S}\left(\delta_{s}^{\gamma}, \omega_{S}\right)\right)$ to $\operatorname{Coker}\left(\operatorname{Hom}_{S}\left(\theta, \omega_{S}\right)\right)$ which yields to

$$
-\operatorname{indeg}\left(\operatorname{Coker}\left(\operatorname{Hom}_{S}\left(\theta, \omega_{S}\right)\right)_{(*, 0)}\right) \leq-\operatorname{indeg}\left(\operatorname{Coker}\left(\operatorname{Hom}_{S}\left(\delta_{s}^{\gamma}, \omega_{S}\right)\right)_{(*, 0)}\right) .
$$


Thus to get an upper bound for the regularity, we need to estimate the latter initial degree. According to the above-mentioned construction of $D_{r+s-1}$ and $\omega_{S}$, we have

$$
\begin{gathered}
\operatorname{Hom}_{S}\left(D_{r+s-1}, \omega_{S}\right) \\
=\operatorname{Hom}_{S}\left(\bigoplus_{i=1}^{g-1} S\left(-\sum_{t=1}^{r} i_{t}-\sigma(\mathfrak{a}),-s-r+i\right), \omega_{R}\left[T_{1}, \cdots, T_{r}\right]\left(-\sum_{t=1}^{r} i_{t},-r\right)\right) \\
=\operatorname{Hom}_{S}\left(\bigoplus_{i=1}^{g-1} S, \omega_{R}\left[T_{1}, \cdots, T_{r}\right]\right)(\sigma(\mathfrak{a}), s-i) \\
=\bigoplus_{i=1}^{g-1} \operatorname{Hom}_{S}\left(S, \omega_{R}\left[T_{1}, \cdots, T_{r}\right]\right)(\sigma(\mathfrak{a}), s-i) .
\end{gathered}
$$

Notice that $\operatorname{Hom}_{S}\left(\delta_{s}^{\gamma}, \omega_{S}\right)$ is in fact the first homomorphism in the Koszul complex $\operatorname{Hom}_{S}\left(K \bullet(\gamma ; S), \omega_{S}\right)$. Therefore

$$
\begin{aligned}
\operatorname{Coker}\left(\operatorname{Hom}_{S}\left(\delta_{s}^{\gamma}, \omega_{S}\right)\right)_{(*, 0)}=\bigoplus_{i=1}^{g-1}\left(\frac{\omega_{R}\left[T_{1}, \cdots, T_{r}\right]}{(\gamma) \omega_{R}\left[T_{1}, \cdots, T_{r}\right]}(\sigma(\mathfrak{a}), s-i)\right)_{(*, 0)} \\
=\bigoplus_{i=1}^{g-1}\left(\omega_{R}(\sigma(\mathfrak{a}), 0) \bigotimes_{R} \frac{S}{(\gamma)}(0, s-i)\right)_{(*, 0)} \\
=\bigoplus_{i=1}^{g-1}\left(\omega_{R}(\sigma(\mathfrak{a}), 0) \bigotimes_{R}\left(\frac{S}{(\gamma)}\right)_{(*, s-i)}\right) .
\end{aligned}
$$

At the moment, let $i_{n}=\operatorname{indeg}(I / \mathfrak{a})$. In this case for all $i<i_{n}, I_{i}=\mathfrak{a}_{i}$; thus $T_{1}, \cdots, T_{n-1} \in(\gamma)$ so that

$$
\left(\frac{S}{(\gamma)}\right)_{(*, s-i)}=\bigoplus_{\alpha_{n}+\cdots+\alpha_{r}=s-i}\left(\frac{(\gamma)+R T_{n}^{\alpha_{n}} \ldots T_{r}^{\alpha_{r}}}{(\gamma)}\right) .
$$

It then follows that

$$
\begin{aligned}
& \text { indeg }\left(\operatorname{Coker}\left(\operatorname{Hom}_{S}\left(\delta_{s}^{\gamma}, \omega_{S}\right)\right)_{(*, 0)}\right) \\
& =\operatorname{indeg}\left(\bigoplus_{i=1}^{g-1}\left(\omega_{R}(\sigma(\mathfrak{a}), 0) \bigotimes_{R}\left(\frac{S}{(\gamma)}\right)_{(*, s-i)}\right)\right) \\
& =\min _{i=1}^{g-1}\left\{\operatorname{indeg}\left(\omega_{R}(\sigma(\mathfrak{a}), 0) \bigotimes_{R}\left(\frac{S}{(\gamma)}\right)_{(*, s-i)}\right)\right\} \\
& \geq \operatorname{indeg}\left(\omega_{R}(\sigma(\mathfrak{a}))\right)+\min _{i=1}^{g-1}\left\{\operatorname{indeg}\left(\bigoplus_{\alpha_{n}+\cdots+\alpha_{r}=s-i}\left(\frac{(\gamma)+R T_{n}^{\alpha_{n}} \ldots T_{r}^{\alpha_{r}}}{(\gamma)}\right)\right)\right\} \\
& \geq \operatorname{indeg}\left(\omega_{R}(\sigma(\mathfrak{a}))\right)+(s-g+1) i_{n} \\
& \geq-\operatorname{reg}(R)+d-\operatorname{dim}\left(R_{0}\right)-\sigma(\mathfrak{a})+(s-g+1) \operatorname{indeg}(I / \mathfrak{a}),
\end{aligned}
$$

where the last inequality follows from Proposition 3.4. It now follows from (3.1), (3.2), and (3.3) that

$$
\begin{aligned}
& \operatorname{end}\left(H_{\mathfrak{m}}^{d-s}(R / J)\right) \leq-\operatorname{indeg}\left(\operatorname{Coker}\left(\operatorname{Hom}_{S}\left(\delta_{s}^{\gamma}, \omega_{S}\right)\right)_{(*, 0)}\right) \\
& \leq \operatorname{reg}(R)-d+\operatorname{dim}\left(R_{0}\right)+\sigma(\mathfrak{a})-(s-g+1) \operatorname{indeg}(I / \mathfrak{a}) .
\end{aligned}
$$


Again, according to Proposition 3.4 we have $\operatorname{reg}(R / J) \leq \operatorname{end}\left(H_{\mathfrak{m}}^{d-s}(R / J)\right)+d-s$, which, in conjunction with the above inequality, implies that

$$
\operatorname{reg}(R / J) \leq \operatorname{reg}(R)+\operatorname{dim}\left(R_{0}\right)+\sigma(\mathfrak{a})-(s-g+1) \operatorname{indeg}(I / \mathfrak{a})-s .
$$

We recall that in the case of linkage, that is when $s=g$, if in addition one has $\operatorname{dim}\left(R_{0}\right)=0$, then the inequality in Theorem 3.6 is in fact an equality. However, when $\operatorname{dim}\left(R_{0}\right) \neq 0$, the next simple example shows that, in some cases, the regularity of residual intersections (or even linked ideals) may be strictly less than the proposed formula.

Example 3.7. Let $R_{0}:=\mathbb{K}[x]_{(x)}$ and $R:=R_{0}[y]$. In this case let $I=(y)$, $\mathfrak{a}=(x y)$ and $J=(x)$ be ideals of $R$. It is now easy to see that $I$ is linked to $J$ by $\mathfrak{a}$, whence the invariants mentioned in Theorem 3.6 are determined as follows: $\operatorname{reg}(R)=\operatorname{reg}(R / I)=\operatorname{reg}(R / J)=0, \operatorname{dim}\left(R_{0}\right)=1, \sigma(\mathfrak{a})=1, s-g+1=1$, $\operatorname{indeg}(I / \mathfrak{a})=1$, and indeg $(J / \mathfrak{a})=0$. Therefore the formula is the equality for $R / J$ and a strict inequality for $R / I$.

3.1. Graded canonical module of residual intersections. In this section, assume in addition that $R=\bigoplus_{i=0}^{\infty} R_{i}$ is a standard positively graded Noetherian ring over an Aritinian local ring $\left(R_{0}, \mathfrak{m}_{0}\right)$ with infinite residue field. As well, the ideals $\mathfrak{a}, I$, and $J$ are assumed to be homogeneous.

To get a graded version of Artin-Nagata's key lemma [1, Lemma 2.3], we need to fix the following convenience.

Definition 3.8. Suppose that $J=\mathfrak{a}: I$ is an (geometric) $s$-residual intersection of $I$. We say that $\mathfrak{a}$ has an $A-N$ homogeneous generating set if there exists a homogeneous generating set $a_{1}, \cdots, a_{s}$ of $\mathfrak{a}$ such that $\left(a_{1}, \cdots, a_{i}\right): I$ is an (geometric) $i$-residual intersection of $I$ for all $s \geq i \geq g$.

As an example of an A-N homogeneous generating set, we shall see in Lemma 3.11 that if $I$ is a homogeneous ideal of the $\mathrm{CM} \operatorname{ring} R$ which satisfies $G_{\infty}$, then for any residual intersection $\mathfrak{a}: I$ of $I, \mathfrak{a}$ has an A-N homogeneous generating set.

The following lemma is needed in the proof of Lemma 3.11. We recall that a proof of this lemma in the (non-graded) local case is given in [24, Theorem 5.8] and [30, Lemma 1.3], but the proof in the local case cannot be applied in the graded case. Also [6, Lemma 2.5] details what one can imagine about Lemma 3.11. However, for the proof of [6, Lemma 2.5] a proof of Lemma 3.9 seems to be indispensable.

Lemma 3.9. Let $M=\bigoplus_{j \in \mathbb{Z}} M_{i}$ be a finitely generated graded $R$-module minimally generated by homogeneous elements of degrees $d_{1} \geq \cdots \geq d_{s}$. Then for any finite set of homogeneous prime ideals $\mathcal{P}=\left\{\mathfrak{p}_{1}, \cdots, \mathfrak{p}_{\mathfrak{n}}\right\}$, there exists a homogeneous element $x \in M$ of degree $d_{1}$ such that for all $1 \leq i \leq n, \mu\left((M /(R x))_{\mathfrak{p}_{\mathfrak{i}}}\right)=\max \left\{0, \mu\left((M)_{\mathfrak{p}_{\mathfrak{i}}}\right)-\right.$ $1\}$.

Proof. We first note that for a graded $R$-module $L$ and a homogeneous prime ideal $\mathfrak{p}, \mu\left(L_{(\mathfrak{p})}\right)=\mu\left(L_{\mathfrak{p}}\right)$, where $L_{(\mathfrak{p})}$ is the homogeneous localization of $L$ at $\mathfrak{p}$. Therefore in the course of the proof we deal with the homogeneous localization instead of the usual localization. We may also assume that $(M)_{\mathfrak{p}_{\mathfrak{i}}} \neq 0$ for every $1 \leq i \leq n$. Let $M^{i}$ be the preimage of $\mathfrak{p}_{\mathfrak{i}} M_{\left(\mathfrak{p}_{\mathfrak{i}}\right)}$ in $M$. Our aim is to show that $M_{d_{1}} \backslash \bigcup_{i=1}^{n}\left(M^{i}\right) \neq \emptyset$.

Let $\left\{m_{1}, \cdots, m_{l}\right\}$ be a homogeneous minimal generating set of $M$ with $\operatorname{deg} m_{i}=$ $d_{i}$. If $\mathfrak{m} \in \mathcal{P}$, say $\mathfrak{m}=\mathfrak{p}_{1}$, then $m_{1} \notin M^{1}$, since $m_{1}, \cdots, m_{l}$ is a minimal generating set of $M$. For another element $\mathfrak{p}_{i} \in \mathcal{P} \backslash\{\mathfrak{m}\}$, as $M \neq M^{i}$, there exists $i_{j} \in\{1, \cdots, s\}$ 
such that $m_{i_{j}} \notin M^{i}$. Hence if we set $c_{i}=d_{1}-d_{i_{j}}$, since $\left(R_{0}, \mathfrak{m}_{0}\right)$ is Artinian and $R$ is a standard positively graded ring, we have $R_{c_{i}} \backslash\left(\mathfrak{p}_{i}\right)_{c_{i}} \neq \emptyset$. Now for any $r_{i} \in R_{c_{i}} \backslash\left(\mathfrak{p}_{i}\right)_{c_{i}}, r_{i} m_{i_{j}} \in M_{d_{1}} \backslash M^{i}$.

Therefore for all $1 \leq i \leq n, M_{d_{1}} \neq M_{d_{1}}^{i}$; in particular, by NAK's lemma, $M_{d_{1}} \neq M_{d_{1}}^{i}+\mathfrak{m}_{0} M_{d_{1}}$. Now taking into account that $R_{0} / \mathfrak{m}_{0}$ is an infinite field, we have $M_{d_{1}} \neq \bigcup_{i=1}^{n}\left(M_{d_{1}}^{i}+\mathfrak{m}_{0} M_{d_{1}}\right)$. In particular, $M_{d_{1}} \backslash \bigcup_{i=1}^{n} M_{d_{1}}^{i} \neq \emptyset$, as desired.

Remark 3.10. Keep the same assumptions as in Lemma 3.9.

(i) If $\mathfrak{m} \notin \mathcal{P}$, then for any $d \geq d_{1}$ there exists an element of degree $d$ that satisfies the assertion of the lemma. Indeed, in this case, for any $\mathfrak{p} \in \mathcal{P}$ and $c \geq 0, R_{c} \neq \mathfrak{p}_{c}-$ the fact which is needed for the proof.

(ii) If $\left(R_{0}, \mathfrak{m}_{0}\right)$ is not Artinian, then Lemma 3.9 is no longer true. As a counterexample, suppose that $\left(R_{0}, \mathfrak{m}_{0}\right)$ is a Noetherian local ring and that $\mathfrak{p}$ and $\mathfrak{q}$ are two prime ideals of $R_{0}$, not one contained in the other. Let $X$ be an indeterminate. Consider $M=R_{0} / \mathfrak{p} \oplus R_{0} / \mathfrak{q}(-1)$ as a graded $R=R_{0}[X]$ module by trivial multiplication. Under these circumstances for $\mathcal{P}=\{\mathfrak{p}+$ $(X), \mathfrak{q}+(X)\}$, there exists no appropriate $x$ desired by the lemma.

Lemma 3.11. If $I$ satisfies $G_{s}$ and $J=\mathfrak{a}: I$ is an s-residual intersection of $I$, then $\mathfrak{a}$ has an $A-N$ homogeneous generating set.

Proof. Applying Lemma 3.9 with $\mathcal{P}=\{\mathfrak{m}\}$, the proof is similar to that of [30, 1.4]. We only replace Lemma 1.3 in the proof of [30, Lemma 1.4] with Lemma 3.9 and note that the set $Q$ employed in [30, 1.4] consists entirely of homogeneous prime ideals.

The next lemma is the base in the inductive construction of the canonical module of residual intersections. A proof of this lemma in the local case is given in [19, 2.1] or [30,2.1] and in the graded case in [23, Lemma 2.3].

Lemma 3.12. Let $R$ be $C M$ and let $\omega_{R}$ be its canonical module. Let $I$ be a homogeneous ideal of height $g$ such that $\omega_{R} / I \omega_{R}$ is CM and let $\alpha=\alpha_{1}, \cdots, \alpha_{g}$ be a maximal homogeneous regular sequence in $I$. Set $J=(\alpha): I$. Then $R / J$ is $C M$ and $\omega_{R / J}=\frac{I \omega_{R}}{(\alpha) \omega_{R}}(\sigma((\alpha)))$.

Remark 3.13. Recall that if $I$ is SCM, then $\omega_{R} / I \omega_{R}$ and all of the Koszul homology modules of $\omega_{R}$ with respect to any generating set of $I$ is CM (see for example [12, 2.3.9] or [28]).

Proposition 3.14. Assume $R$ is standard graded Gorenstein over an Artinian local ring $R_{0}$ with the graded canonical module $\omega_{R}=R(b)$, where $b$ is an integer. Suppose that $J=\mathfrak{a}: I$ is a geometric s-residual intersection of $I$. Assume moreover that $I$ is $S C M$ and satisfies $G_{s}$. Then $R / J$ is $C M$ of dimension $d-s$ and $\omega_{R / J} \cong$ $(I+J / J)^{s-g+1}(b+\sigma(\mathfrak{a}))$.

Proof (see [19, 2.3]). The proof proceeds along the same line as in the local case.

Now we are ready to present the second proof of the regularity's inequality of residual intersections. A third proof in the case where $R$ is a polynomial ring over 
a field can be drawn from the minimal free resolution for residual intersections of SCM ideals which satisfy the $G_{s}$ condition presented by A. Kustin and B. Ulrich in $[23,2.1]$.

Proposition 3.15. Assume that $R$ is standard graded Gorenstein, with $R_{0}$ Artinian local, and that $I$ is an SCM ideal which satisfies the $G_{s}$ condition. If $J$ is a geometric s-residual intersection of $I$, then

$$
\operatorname{reg}(R / J) \leq \operatorname{reg}(R)+\sigma(\mathfrak{a})-(s-g+1) \operatorname{indeg}(I / \mathfrak{a})-s,
$$

and the equality holds if and only if $\left((I / \mathfrak{a})_{i}\right)^{s-g+1} \neq 0$, where $i=\operatorname{indeg}(I / \mathfrak{a})$.

Proof. We first recall that, since $\left(R_{0}, \mathfrak{m}_{0}\right)$ is an Artinian local ring, for any $R$-module $L$ and any integer $i, H_{\mathfrak{m}}^{i}(L)=H_{R_{+}}^{i}(L)$. Hence, by the definition of CastelnuovoMumford regularity and Theorem 2.11]

$$
\operatorname{reg}(R / J)=\max \left\{\operatorname{end}\left(H_{R_{+}}^{i}(R / J)\right)+i: 0 \leq i \in \mathbb{Z}\right\}=\operatorname{end}\left(H_{R_{+}}^{d-s}(R / J)\right)+d-s .
$$

Using the graded local duality theorem [2, 13.4 .2 and 13.4.5(iv)], one has $\operatorname{end}\left(H_{R_{+}}^{d-s}(R / J)\right)=\operatorname{end}\left(\operatorname{Hom}_{R_{0}}\left(\omega_{R / J}, E_{R_{0}}\left(R_{0} / \mathfrak{m}_{0}\right)\right)\right)=-\operatorname{indeg}\left(\omega_{R / J}\right)$. Therefore we have to compute indeg $\left(\omega_{R / J}\right)$. By Proposition 3.14 indeg $\left(\omega_{(R / J)}\right)=$ indeg $\left((I+J / J)^{s-g+1}(b+\sigma(\mathfrak{a}))\right)$. Recall that under the conditions of the theorem $I \cap J=\mathfrak{a}$, we have indeg $\left((I+J / J)^{s-g+1}(b+\sigma(\mathfrak{a}))\right)=\operatorname{indeg}\left((I / \mathfrak{a})^{s-g+1}\right)-(b+\sigma(\mathfrak{a})) \geq$ $(s-g+1) \operatorname{indeg}(I / \mathfrak{a})-b-\sigma(\mathfrak{a})$. Thus we get $\operatorname{reg}(R / J) \leq \sigma(\mathfrak{a})-(s-g+1) \operatorname{indeg}(I / \mathfrak{a})+$ $b-s=\operatorname{reg}(R)+\sigma(\mathfrak{a})-(s-g+1) \operatorname{indeg}(I / \mathfrak{a})-s$. Also, the equality holds if and only if indeg $\left((I / \mathfrak{a})^{s-g+1}\right)=(s-g+1) \operatorname{indeg}(I / \mathfrak{a})$.

3.2. Perfect ideals of height 2. It is known that the residual intersection of perfect ideals of height 2 is a determinant ideal so that the Eagon-Northcott complex will provide a free resolution (not necessarily minimal) for such residual intersections. A careful study of shifts in the Eagon-Northcott complex enables us to compute the regularity of residual intersections in this case.

In what follows, $R$ is a standard graded Cohen-Macaulay ring over a field $R_{0}=$ $\mathbb{K}, I$ is a homogeneous ideal of $R$ minimally generated by $f_{1}, \cdots, f_{r} \in R$ with $\operatorname{deg} f_{j}=i_{j}$ for $1 \leq j \leq r$ and also $i_{1} \geq \cdots \geq i_{r-u}>i_{r-u+1}=\cdots=i_{n}$ for some $1 \leq u \leq r$. Let $\mathfrak{a}=\left(l_{1}, \cdots, l_{s}\right)$ be a homogeneous $s$-generated ideal of $R$ properly contained in $I$ with $\operatorname{deg} l_{i}=a_{i}$ for $1 \leq i \leq s$ and $a_{1} \geq \cdots \geq a_{k}>a_{k+1}=\cdots=$ $a_{s}=i_{r}$. Let $J=\mathfrak{a}: I$ be an $s$-residual intersection of $I$.

Theorem 3.16. With the same token as above, if I is a perfect ideal of height 2, then

(i) $J$ is perfect of height $s$,

(ii) $s-k \leq u$,

(iii) $\operatorname{reg}(R / J)=\operatorname{reg}(R)+\sigma(\mathfrak{a})-(s-1) \operatorname{indeg}(I)-s$, whenever $s-k \leq u-1$.

Proof. Since grade $(J)=s=(r-1+s)-r+1, J$ is the determinant ideal generated by $r$-minors of the 0 -th homomorphism, $\psi$, in the mapping cone of the chain map over $\mathfrak{a} \hookrightarrow I$ between free resolutions of $\mathfrak{a}$ and $I$ (see [6. Theorem 1.1]). Furthermore, the Eagon-Northcott complex of $\psi$ provides a free resolution for $R / I_{r}(\psi)=R / J$, say

$$
\mathcal{N}_{\bullet}: 0 \rightarrow N_{s-1}[\sigma] \rightarrow \cdots \rightarrow N_{1}[\sigma] \rightarrow N_{0}[\sigma] \rightarrow R \rightarrow R / J \rightarrow 0
$$


where $N_{j}=\left(\operatorname{Sym}_{j}\left(\bigoplus_{t=1}^{r} R\left(-i_{t}\right)\right)^{*} \otimes \bigwedge^{r+j}\left(\bigoplus_{t=1}^{r+s-1} R\left(-c_{t}\right)\right)\right.$, and $c_{1}, \cdots, c_{r+s-1}$ are integers such that $\left\{c_{1}, \cdots, c_{r+s-1}\right\}=\left\{b_{1}, \cdots, b_{r-1}, a_{1}, \cdots, a_{s}\right\}$ with $c_{1} \geq \cdots \geq$ $c_{r+s-1}$ and $\sigma=\sigma(\mathfrak{a})$. The module $N_{j}, 0 \leq j \leq s-1$, is a graded free module generated by elements of degrees $-\left(i_{t_{1}}+\cdots+i_{t_{j}}\right)+\left(c_{k_{1}}+\cdots+c_{k_{r+j}}\right)$ with $t_{1} \leq$ $\cdots \leq t_{j}$ and $k_{1}<\cdots<k_{r+j}$.

Part (i) immediately follows from the convergence of the spectral sequences derived from the double complex $\mathcal{C}_{\mathfrak{m}}^{\bullet} \otimes_{R} \mathcal{N}_{\bullet}$. To see (ii), note that by part (i) ht $(J)=s$; this means that $\mathfrak{a}$ cannot be generated by a less number of generators than $s$. Now since the $\mathbb{K}$-vector spaces $\mathfrak{a}_{i_{r}}$ is a subspace of $I_{i_{r}}$, the former is of dimension $s-k$ while the latter is of dimension $u$ so that $s-k \leq u$. This proves (ii).

To prove (iii), we first introduce two numerical functions $f$ and $n . \quad f(j):=$ $\sum_{t=1}^{r+j} c_{t}-j i_{r}-\sigma=$ The maximum degree of generators of $N_{j}[\sigma]$, and $n(j):=$ The number of generators of $N_{j}[\sigma]$ of the maximum degree $f(j)$.

By the Hilbert-Burch theorem, 9, 3.13], we have $b_{1}, \cdots, b_{r-1}>i_{r} \geq r-1 \geq 1$ and $\sigma=\sum_{t=1}^{r-1} b_{t}$. On the other hand, $f(j+1)-f(j)=c_{r+j+1}-i_{r}$ for $0 \leq j \leq s-2$. Therefore we get the following ordering of $f(j)$ 's for $0 \leq j \leq s-1$ :

$$
0<f(0)<\cdots<f(k-1)=f(k)=\cdots=f(s-1) .
$$

To show the desired formula, it is sufficient to show that $\operatorname{end}\left(H_{\mathfrak{m}}^{d-s}(R / J)\right)=a+e$, where $a=f(s-1)$ and $e=\operatorname{end}\left(H_{\mathfrak{m}}^{d}(R)\right)$.

The first vertical spectral sequence derived from $\mathcal{C}_{\mathfrak{m}} \otimes_{R} \mathcal{N}_{\bullet}$ is the following exact sequence:

$$
0 \rightarrow H_{\mathfrak{m}}^{d-s}(R / J) \rightarrow H_{\mathfrak{m}}^{d}\left(N_{s-1}[\sigma]\right) \rightarrow \cdots \rightarrow H_{\mathfrak{m}}^{d}\left(N_{0}[\sigma]\right) \rightarrow H_{\mathfrak{m}}^{d}(R) \rightarrow 0 .
$$

For $0 \leq j \leq s-1, H_{\mathfrak{m}}^{d}\left(N_{j}[\sigma]\right)=\cdots \oplus\left(H_{\mathfrak{m}}^{d}(R)(-a)\right)^{n(j)}$. Hence if $H_{\mathfrak{m}}^{d}(R)_{e}=\mathbb{K}^{t}$ for some $t>0$, then the $(e+a)$-th strand of (3.3) is

$$
0 \rightarrow H_{\mathfrak{m}}^{d-s}(R / J)_{e+a} \rightarrow \mathbb{K}^{t n(s-1)} \rightarrow \cdots \rightarrow \mathbb{K}^{t n(k-1)} \rightarrow 0 .
$$

Therefore $H_{\mathfrak{m}}^{d-s}(R / J)_{e+a} \neq 0$ if and only if $\sum_{j=k-1}^{s-1}(-1)^{j} n(j) \neq 0$. A straightforward computation shows that $n(j)=\left(\begin{array}{c}s-k \\ j-k+1\end{array}\right)\left(\begin{array}{c}u+j-1 \\ u-1\end{array}\right)$ for $k-1 \leq j \leq s-1$. Consequently, to show that $\sum_{j=k-1}^{s-1}(-1)^{j} n(j) \neq 0$, it is enough to show that $(-1)^{k-1-(s-k)} \beta_{s-k}(t):=\sum_{j=k-1}^{s-1}\left(\begin{array}{c}s-k \\ j-k+1\end{array}\right) j^{t}$ has the same sign for each $0 \leq t \leq$ $u-1$, and at least one of them is non-zero. This follows from the assumption $s-k \leq u-1$.

Remark 3.17. In the case where $s-k=u$, the definition of the numerical function $\beta$ in the above proof implies that $\beta_{u}(t)=0$ for all $t \leq u-1$, which means that $\sum_{j=k-1}^{s-1}(-1)^{j} n(j)=0$. Thus $\operatorname{reg}(R / J)<\operatorname{reg}(R)+\sum_{i=1}^{s} d_{i}-(s-1) i_{r}-s$. Indeed in this case if we have $i_{1} \geq \cdots \geq i_{r-v}>i_{r-v+1}=\cdots=i_{r-u}>i_{r-u+1}=\cdots=i_{n}$ and $a_{1} \geq \cdots \geq a_{k-t}>a_{k-t+1}=\cdots=a_{k}=i_{r-u}>a_{k+1}=\cdots=a_{s}=i_{r}$, then by the same argument as in the proof of Theorem 3.16. one can see that $t \leq u-v$ and that $\operatorname{reg}(R / J)=\operatorname{reg}(R)+\sigma(\mathfrak{a})-(s-1) i_{r-u}-s$, whenever $t<u-v$.

Continuing in this way, by a similar argument as in Theorem 3.16, one can deduce the next proposition.

Proposition 3.18. If $I$ is a perfect ideal of height 2 and $J \neq R$, then

$$
\operatorname{reg}(R / J)=\operatorname{reg}(R)+\sigma(\mathfrak{a})-(s-1) \operatorname{indeg}(I / \mathfrak{a})-s .
$$




\section{ACKNOWLEDGMENT}

This work was done as a part of the author's Ph.D. thesis as a Cotutelle program between Iran and France. He would like to thank his advisor, Professor Marc Chardin, for suggesting the problem, for his enormous help and for being so supportive. The author would also like to thank the referee for his/her useful comments and careful reading of the manuscript.

\section{REFERENCES}

[1] M. Artin and M. Nagata, Residual intersection in Cohen-Macaulay rings, J. Math. Kyoto Univ. (1972), 307-323. MR0301006 (46:166)

[2] M. Brodmann, R.Y. Sharp, Local Cohomology, An Algebric Introduction with Geometric Application, Cambridge University Press, Cambridge, 1998. MR1613627 (99h:13020)

[3] W. Bruns, J. Herzog, Cohen-Macaulay Rings, revised version, Cambridge University Press, Cambridge, 1998. MR1251956 (95h:13020)

[4] W. Bruns, A. Kustin, and M. Miller, The resolution of the generic residual intersection of a complete intersection, J. Algebra 128 (1990), 214-239. MR.1031918 (91c:13009)

[5] M. Chardin and B. Ulrich, Liaison and Castelnuovo-Mumford regularity, American Journal of Mathematics 124 (2002), 1103-1124. MR1939782 (2004c:14095)

[6] M. Chardin, D. Eisenbud and B. Ulrich, Hilbert functions, residual intersections, and residually $S_{2}$ ideals, Composito Mathematica 125 (2001), 193-219. MR.1815393 (2002g:13034)

[7] C. Cumming, Residual intersections in Cohen-Macaulay rings, J. Algebra 308 (2007), 91106. MR2290911 (2008f:13042)

[8] J. A. Eagon and D. G. Northcott, Ideals defined by matrices and a certain complex associated with them, Proc. Roy. Soc. Ser. A269 (1962), 188-204. MR0142592 (26:161)

[9] D. Eisenbud, The geometry of syzygies, Graduate Texts in Math. 150, Springer, New York, 2005. MR2103875 (2005h:13021)

[10] W. Fulton, Intersection Theory, Springer-Verlag, Berlin, 1998. MR1644323 (99d:14003)

[11] A. Grothendick, EGA III, 11, publications mathèmatique de l'I.H.E.S, 1961.

[12] S. H. Hassanzadeh, Castelnuovo-Mumford regularity of residual intersections and liaison, Ph.D. Thesis, Université Pierre et Marie Curie (Paris VI) and Tarbiat Moallem University of Tehran, 2009.

[13] J. Herzog, E. Kunz, Der kanonische Modul eines Cohen-Macaulay-Rings, Lecture Notes in Math., vol. 238, Springer, 1971. MR0412177 (54:304)

[14] J. Herzog, A. Simis, and W. Vasconcelos, Koszul homology and blowing-up rings, Commutative Algebra (Trento, 1981), Lecture Notes in Pure and Appl. Math., vol. 84, Marcel Dekker, NY (1983), pp. 79-169. MR686942 (84k:13015)

[15] J. Herzog, A. Simis, and W. Vasconcelos, On the arithmetic and homology of algebras of linear type, Trans. Amer. Math. Soc. 283, No. 2 (1984), 661-683. MR737891 (86a:13015)

[16] J. Herzog, W.V. Vasconcelos, and R. Villarreal, Ideals with sliding depth, Nagoya Math. J. 99 (1985), 159-172. MR805087 (86k:13022)

[17] S. Huckaba, C. Huneke, Rees algebras of ideals having small analytic deviation, Trans. Amer. Math. Soc. 339 (1993), no. 1, 373-402. MR1123455 (93k:13008)

[18] C. Huneke, Strongly Cohen-Macaulay schemes and residual intersections, Trans. Amer. Math. Soc. 277(1983), 739-763. MR694386 (84m:13023)

[19] C. Huneke, B. Ulrich, Residual Intersection, J. Reine Angew. Math. 390 (1988), 1-20. MR953673 (89j:13024)

[20] C. Huneke, B. Ulrich, The Structure of Linkage, Ann. of Math. 126 (1987), 277-334. MR.908149 (88k:13020)

[21] E. Hyry The diagonal subring and the Cohen-Macauley property of a multigraded ring, Trans. Amer. Math. Soc. 351 (1999), 2213-2232. MR.1467469 (99i:13005)

[22] A. Kustin and B. Ulrich, A family of complexes associated to an almost alternating map, with applications to residual intersections, Mem. Amer. Math. Soc. 461 (1992). MR.1091668 (92i:13012)

[23] A. Kustin and B. Ulrich, If the socle fits, J. Algebra 147 (1992), 63-80. MR1154674 (93e:13017) 
[24] H. Matsumura, Commutative ring theory, Cambridge University Press, Cambridge, 1986. MR879273(88h:13001)

[25] C. Peskine and L. Szpiro, Liaison des variétés algébriques, Invent. Math. 26 (1974), 271-302. MR0364271 (51:526)

[26] C. Peskine and L. Szpiro, Dimension projective finie et cohomologie locale, Pbl. Math. I.H.E.S 42 (1972), 47-119. MR0374130 (51:10330)

[27] C. Polini, B. Ulrich, A formula for the core of an ideal, Math. Annalen 331 (2005), 487-503. MR2122537 (2006k:13020)

[28] H. Sanders, Cohen-Macaulay properties of the Koszul homology, Manuscripta Math. 55 (1986), 343-357. MR836869 (87g:13020)

[29] N. V. Trung, The largest non-vanishing degree of graded local cohomology modules, J. Algebra 215 (1999), 481-499. MR 1686202 (2000f:13038)

[30] B. Ulrich, Artin-Nagata properties and reduction of ideals, Contemp. Math. 159 (1994), 373-400. MR.1266194 (95a:13017)

[31] W. V. Vasconcelos, Arithmetic of Blowup Algebras, 195, Cambridge University Press, 1994. MR.1275840 (95g:13005)

[32] W. Vasconcelos, Hilbert functions, analytic spread, and Koszul homology, Contemp. Math. 159 (1994), 401-422. MR1266195 (95a:13006)

[33] C. A. Weibel, An Introduction to Homological Algebra, 38, Cambridge University Press, 1994. MR.1269324 (95f:18001)

Faculty of Mathematical Sciences and Computer, Tarbiat Moallem University, 599 Taleghani Avenue, Tehran 15618, Iran - and - Institut de Mathematiques, Université Pierre et Marie Curie, 175 rue du Chevaleret, 75013, Paris, France

Current address: Departamento de Matemática, Universidade Federal de Pernambuco, Av. Jornalista Anibal Fernandes, sn, Cidade Universitária 50740-560, Recife, Pernambuco, Brazil E-mail address: hamid@dmat.ufpe.br 